
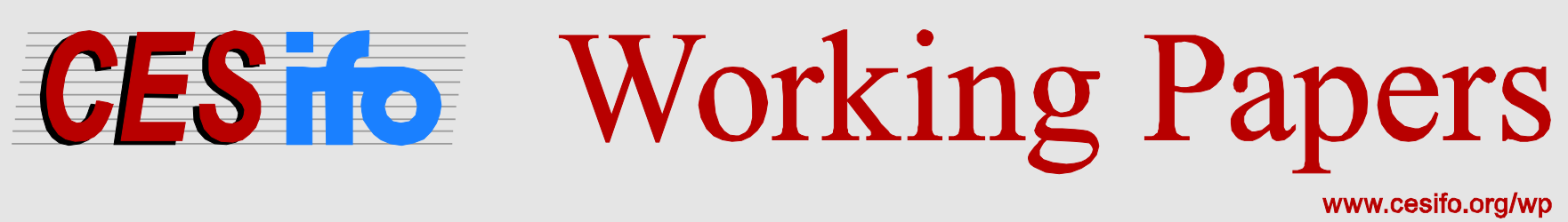

\title{
Net Neutrality and Internet Fragmentation: The Role of Online Advertising
}

\author{
Anna D’Annunzio \\ Antonio Russo
}

CESIFO WORKING PAPER NO. 5467

CATEGORY 11: INDUSTRIAL ORGANISATION

August 2015

An electronic version of the paper may be downloaded

- from the SSRN website:

- from the RePEc website:

- from the CESifo website:

wWw.SSRN.com

Www.RePEc.org

www.CESifo-group.org/wp 


\title{
Net Neutrality and Internet Fragmentation: The Role of Online Advertising
}

\begin{abstract}
We investigate the relation between Net Neutrality regulation and Internet fragmentation. We model a two-sided market, where Content Providers (CPs) and consumers interact through Internet Service Providers (ISPs), and CPs sell consumers' attention to advertisers. Under Net Neutrality, a zero-price rule is enforced. By contrast, in the Unregulated Regime, ISPs make access to their subscribers for CPs conditional on payment of a termination fee. Multiple impressions of an ad on the same consumer are partially wasteful. Thus, equilibrium ad rates decrease when audiences overlap. We show that ISPs may strategically set termination fees to induce fragmentation. This takes place when advertising revenues are potentially large but strongly diminished by competition among CPs, and when consumers are not highly sensitive to content availability. We therefore identify an important link between termination fees, the online advertising market and Internet fragmentation. We extend the model to account for multihoming consumers, vertically integrated ISPs, third-party advertising platforms and heterogeneous CPs.
\end{abstract}

JEL-Code: L100, D430, L130, L510.

Keywords: net neutrality, two-sided markets, internet, advertising, fragmentation.

\author{
Anna D’Annunzio \\ Telenor Research \\ Oslo - Fornebu / Norway \\ anna.dannunzio@telenor.com
}

\author{
Antonio Russo \\ ETH Zurich \\ KOF Swiss Economic Institute \\ LEE G 116, Leonhardstrasse 21 \\ Switzerland - 8092 Zurich \\ russo@kof.ethz.ch
}

This version: July 2015

An earlier version of this paper circulated with the title: "Network Neutrality, Access to Content and Online Advertising”. We thank Bruno Jullien for his important advice. We also thank Charles Angelucci, Marc Bourreau, Carlo Cambini, Jacques Crémer, Raffaele Fiocco, Øystein Foros, Gorm Grønnevet, Bjørn Hansen, Carole Haritchabalet, Heiko Karle, Marko Köthenbürger, Jan Krämer, Yassine Lefouili, Pierfrancesco Reverberi, Wilfried Sand-Zantman, Paul Seabright, Lars Sørgard and the audiences at CRESSE 2013, EARIE 2013 and NHH Bergen for helpful comments and discussions. Finally, we thank the Editor, Giacomo Calzolari, and two anonymous Referees for valuable comments that greatly helped improve the paper. All errors are ours alone. The views expressed in this paper are those of the authors only and not those of Telenor. The Supplementary Appendix to this paper is available on the authors' website. 


\section{Introduction}

Traditionally, the Internet has been characterized by the Net Neutrality principle. This principle has various practical implications. In particular, it implies a zero-price and a nondiscrimination rule (Schuett, 2010). The former establishes that Internet Service Providers (hereafter, ISPs) should not collect fees from Content Providers (hereafter, CPs) for delivering (or "terminating") data to final users, while the latter establishes that ISPs should treat all traffic equally. ${ }^{1}$ Presently, there is a very important policy debate on whether Net Neutrality should be codified in formal regulation. Opponents assert that allowing ISPs to have greater pricing flexibility will ensure a more efficient use of bandwidth and strengthen investment in network infrastructure. Proponents argue instead that Net Neutrality regulation is necessary to preserve plurality on the Internet, alongside content innovation (Krämer, Wiewiorra and Weinhardt, 2013).

The above issues have received considerable attention in previous literature (reviewed in Section 2 below). There is, however, another aspect of the Net Neutrality debate that has been much less scrutinized thus far. Namely, the implications of a zero-price rule for Internet fragmentation. Several scholars, regulators and Internet operators have expressed concerns that termination fees could lead to a fragmented network, with some consumers being unable to access content available to others, thereby creating "multiple Internets" (Lee and Wu, 2009). ${ }^{2}$ Despite this, economic research has largely neglected the problem.

Our objective is to shed some light on this important, yet unexplored, issue. We do so by means of a simple model, considering two CPs and two ISPs. An ISP is a platform connecting CPs to consumers and a CP is a platform selling consumers' attention to advertisers. Consumers pay the ISP for a connection to the Internet and browse content free of charge.

\footnotetext{
${ }^{1}$ Currently, the general practice is that CPs pay a fee to the ISP that connects them to the Internet, but they do not pay ISPs to terminate their traffic to end users. However, in 2013 French ISP Orange reportedly asked Google to pay for delivering its traffic (see http://www.forbes.com/sites/ewanspence/2013/01/20/whyoranges-dominance-in-africa-forced-google-to-pay-for-traffic-over-their-mobile-network) and in February 2014 Netflix agreed to pay Comcast for peering, resulting in improved service to Comcast's subscribers (see http://online.wsj.com/news/articles/SB10001424052702304834704579401071892041790).

${ }^{2}$ The FCC recently adopted the "Open Internet Rules", stating that ISPs "shall not block lawful content, applications, services, or non-harmful devices, subject to reasonable network management" (FCC, 2015). Furthermore, the European Commission stated that ISPs should not "block or throttle traffic in their networks or give priority to some particular content or services in exchange of payment" (http://europa.eu/rapid/pressrelease_MEMO-15-5275_en.htm). In April 2014, Neelie Kroes, European Commissioner for the Digital Agenda, stated: "the Internet is unified, global, open. [...] Fail to act, fail to reform - and we could see the net fragment" (neurope.eu, 2014, "Kroes: Defending open internet and net neutrality" http://www.neurope.eu/article/kroes-defending-open-internet-and-net-neutrality). Content Providers such as Netflix have repeatedly called for regulation preventing ISPs from charging for termination. As a matter of fact, several countries such as the Netherlands and Chile, have enshrined Net Neutrality principles into legislation, banning termination fees.
} 
We consider two regulatory regimes. Under Net Neutrality, a zero-price rule is enforced: ISP have to terminate all traffic free of charge. In the Unregulated Regime, on the other hand, CPs can reach subscribers of an ISP only on condition that they pay a termination fee.

It is well recognized that the Internet is a two-sided market bringing together consumers and advertisers. Yet, to the best of our knowledge, previous literature on Net Neutrality has never explicitly studied the advertising side. The main novelty of our approach is that we model competition in such market. We account for the stylized fact that the marginal value of an ad decreases with the number of times a consumer is exposed to it (see, e.g., Calvano and Jullien, 2011; Anderson, Foros and Kind, 2014; Athey, Calvano and Gans, 2013; Ambrus and Reisinger, 2006). Given that consumers commonly consult several online contents in a short time frame, an advertiser's willingness to pay for ad impressions diminishes when audiences overlap. ${ }^{3}$

We show that this mechanism is at the heart of the forces driving network fragmentation. Indeed, a rational response by CPs would be to avoid competition by serving different audiences. However, under Net Neutrality, neither ISPs nor CPs are able to shape the configuration of the network. As a result, content is available to all consumers (a situation referred to as "Universal Connection"). Things are different in the Unregulated Regime, as termination fees create a link between ISPs and advertising. Because they can recover CP profits via termination fees, ISPs behave as editors, caring about the profitability of the content they carry. Hence, they have an incentive to induce fragmentation when (i) advertising revenues are potentially large but strongly diminished by competition among CPs, and (ii) contents are not highly valuable and complementary for consumers. Fragmentation, we show, can be achieved by raising termination fees to a level high enough for a CP to be willing to pay only as long as it has no competition for consumers' attention. Nonetheless, Universal Connection still emerges when competition has limited impact on advertising profits (so that little gain can be expected by creating exclusive audiences) and when content is highly valuable to consumers (so that offering additional content boosts demand for an ISP's services).

In summary, our results suggest that Net Neutrality helps preserve universal availability of online content. If the Unregulated Regime is adopted instead, the extent to which repeated ad impressions lose value is crucial in shaping the network configuration. We therefore contribute to the current debate by uncovering an important relation between Net Neutrality, Internet fragmentation and competition on the online advertising market. We believe this should not be ignored when drafting regulations on these critical issues.

\footnotetext{
${ }^{3}$ Todd Haskell, vice-president of advertising for the New York Times, stated that "rates for online-video ads have not been increasing though publishers have more demand than supply". He also stated that publishers may seek differentiation from competitors as a way to "avoid the downward commoditized price positioning" of ad slots (WSJ.com, 2013, "If Media's Future Is Online, Where Are the Profits?", retrieved June 2013).
} 
For ease of exposition and in order to simplify the analytics, our baseline model imposes a restriction on how termination fees are set. Namely, we assume that each ISP charges all CPs the same fee (we refer to this as "uniform fees"). We then relax this assumption in an extension, allowing for discriminatory termination fees. We show that discriminatory fees strenghten our basic arguments. We propose further extensions in the final part of the paper: we consider heterogeneous CPs, alternative arrangements for the sale of ad impressions (i.e. outsourcing to advertising platforms either run by third parties or by ISPs), multi-homing consumers, vertical integration of content and Internet service providers. Finally, we discuss alternative timings of our model.

The remainder of this paper is organized as follows. Section 2 presents a brief literature review. In Section 3 we describe the baseline model, solved in Section 4. Section 5 contains the extensions. Section 6 concludes. Unless otherwise stated, Proofs of Propositions and Lemmas are in Appendix. Furthermore, a Supplementary Appendix (available on the authors' website) contains formal derivations of additional results that we only discuss in the main text.

\section{Literature}

There is a wide debate on Net Neutrality that has only recently been analyzed from an economic perspective. ${ }^{4}$ The main focus of previous literature has been on service tiering and investment. Hermalin and Katz (2007) study the desirability of traffic discrimination, finding an ambiguous welfare comparison between the Unregulated Regime and Net Neutrality. Choi and Kim (2010) consider service tiering and investment incentives for a monopolist ISP and for CPs in different regulatory regimes. The effect of the regulatory regime on investment and social welfare is again ambiguous. Bourreau, Kourandi and Valletti (2015) study a similar issue in a model with two competing ISPs. Under discrimination, ISPs have larger investment incentives, there are more active content providersand there is less congestion. Hence, the discriminatory regime is welfare superior to Net Neutrality. Economides and Hermalin (2012) show that the socially optimal configuration maximizes contents delivered to consumers. Differently from Bourreau, Kourandi and Valletti (2015), the discriminatory regime can either increase or decrease the variety of distributed content. Contrary to Choi and Kim (2010), abandoning Net Neutrality increases the incentives to invest in infrastructure. However, the net effect on welfare is once again ambiguous.

Unlike the authors of the above papers, we consider Net Neutrality as a zero-price rule: it requires that ISPs charge CPs no fees in order to terminate traffic to final users. This

\footnotetext{
${ }^{4}$ See Lee and $\mathrm{Wu}(2009)$ for a discussion on the economic issues concerning Net Neutrality. See Schuett (2010) and Krämer, Wiewiorra and Weinhardt (2013) for a review of the literature.
} 
definition is also used in Economides and Tag (2012), who concentrate on pricing issues linked to the two-sidedness of the market that arise due to a departure from Net Neutrality. Musacchio, Schwartz and Walrand (2009) analyze a similar issue in a model where ISPs and CPs can also invest in network quality.

As mentioned in the introduction, previous papers on Net Neutrality treat profitability of content providers as exogenous. In our model it results instead from platform competition on the advertising market. A novelty of our work is therefore that it is at the intersection between the literature on Net Neutrality and that on online advertising markets. Ambrus, Calvano and Reisinger (2013) and Anderson, Foros and Kind (2014) build models of media platform competition where both consumers and advertisers multi-home. A common finding is that platforms have monopoly power over single-homing consumers, but can only charge the incremental value of ad impressions for multi-homing consumers. Our model captures the same key effect. ${ }^{5}$

Furthermore, our paper is among the very first to analytically study the link between termination fees and Internet fragmentation. In a recent paper, Kourandi, Krämer and Valletti (2015) also consider this question. The most important differences between our approach and theirs are that their main focus is on exclusive contracts (termination fees are exogenous in their framework) and that they do not explicitly model the advertising market. Thus, our work investigates the link between pricing regulation and Internet fragmentation in a complementary way.

Our work relates to the theory of vertical restraints. In our baseline model, each ISP can be thought of as a monopolist upstream firm that sells CPs access to its subscribers' eyeballs. ${ }^{6}$ CPs can be thought of as downstream firms, (re)selling those eyeballs to advertisers. As previous literature has pointed out (see, e.g., Rey and Vergé, 2008, and Rey and Tirole, 2007), an upstream firm can be better off reducing downstream competition if it can appropriate some of the downstream industry profit. Similar forces are at work in our model: foreclosing a CP may boost the profits of the remaining one, which the ISP can recoup through the termination fee. Importantly, though, incentives to foreclose depend on the contractual arrangements linking different sides of the market (we investigate this last point in Section 5.2 below) ${ }^{7}$

Finally, our results are related to the recent contribution by de Cornière and Taylor

\footnotetext{
${ }^{5}$ Gal-Or and Dukes (2003) show that commercial media have incentives to minimally differentiate in order to induce less informative advertising by producers. This weakens competition on the product market, enabling media to increase ad rates.

${ }^{6}$ In the terminology introduced by Armstrong (2006), our ISPs are "competitive bottlenecks".

${ }^{7}$ It should also be noted that, in our model, ISPs are two-sided platforms. We find that a necessary condition for foreclosure is that demand on the consumer side is relatively insensitive to content availability.
} 


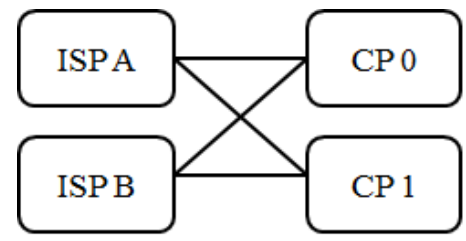

Universal Connection

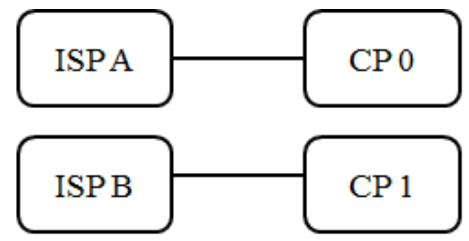

Total Fragmentation

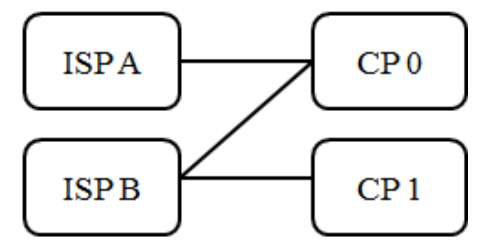

Partial Fragmentation

Figure 1: Main network configurations.

(2014). They find that a mainstream search engine may have incentives to alter competition among CPs on the advertising market. By biasing search results, the engine may increase the portion of advertising revenues it retains.

\section{The Basic Model}

\subsection{Setup}

We consider two ISPs, indexed by $i=A, B$, and two CPs, indexed by $j=0,1$. In our model, an ISP is a platform connecting consumers to CPs, and a CP is a platform connecting consumers to advertisers. ISPs and CPs are independent firms. Our focus is on pricing decisions by residential ISPs regarding the "last mile" connection service to consumers. Accordingly, we take as given the existence of a backbone that connects $A$ and $B$ to the entire network and the connection arrangements whereby CPs access the Internet. ${ }^{8}$

Throughout the paper, we will use the shorthand "CP $j$ connects to ISP $i$ " to mean that $\mathrm{CP} j$ has its traffic terminated on ISP $i$ 's network (hence, $j$ 's content reaches $i$ 's subscribers). For future reference, it is useful to introduce some terminology concerning the main network configurations we will discuss in this paper. We will refer to the configuration where all CPs connect to all ISPs as "Universal Connection". We will refer to the configuration where each CP connects to a different ISP as "Total Fragmentation". Finally, a configuration where a CP connects to one ISP, while the other CP connects to both is referred to as "Partial Fragmentation". Figure 1 provides an illustration.

We are interested in the implications of allowing ISPs to charge CPs for delivering content

${ }^{8}$ The Internet is an interconnected "network of networks" and packets of content usually travel across several networks before reaching their destination. At the risk of excessive simplification, we can summarize the structure of contracts underpinning the Internet as follows: consumers acquire access from a residential ISP (say, Comcast); CPs (say, Google) also acquire access to the Internet via some ISP. In turn, ISPs make peering and transit agreements with each other and with backbone ISPs, which connect them (and their customers) to the entire Internet. In line with previous literature (Choi and Kim, 2010; Economides and Tag, 2012; Economides and Hermalin; 2012) we take the existence of transit and peering agreements between ISPs as given. 
over the last mile connection. To this end, we consider two regulatory regimes: one in which Net Neutrality regulation is in place and one in which it is not, referred to as the Unregulated Regime. In the former, ISPs have to terminate all traffic free of charge. We therefore model Net Neutrality as a zero-price rule on one side of the market (see, e.g., Schuett, 2010), as ISPs are only permitted to charge end consumers. By contrast, in the Unregulated Regime, termination of traffic generated by CPs is conditional on payment of a fee.

Internet Service Providers. ISPs are located at the extremes of a Hotelling line: ISP $A$ is located at point 0 and ISP $B$ at point $1 .^{9}$ ISP $i$ sets a subscription fee $a_{i}$ for consumers who want to join its network. In the Unregulated Regime, ISP $i$ also sets a non-contingent termination fee $F_{i}$ that CPs have to pay in order to reach $i$ 's consumers. There is no restriction on the sign of termination fees. ${ }^{10} F_{i}$ is exclusionary: CP $j$ can connect to ISP $i$ only by paying it. In the Net Neutrality regime, $F_{i}=0$ for any $i$. The profit function of ISP $i$ is

$$
\pi_{i}=a_{i} q_{i}+\sum_{j=0,1} F_{i} I_{i j}, \quad i=A, B \quad j=0,1
$$

where $q_{i}$ is the quantity of consumers subscribing to ISP $i$ and $I_{i j}$ is an indicator function, such that $I_{i j}=1$ if $\mathrm{CP} j$ connects to ISP $i$ and $I_{i j}=0$, otherwise. Note that, in our baseline model, termination fees are uniform, i.e. they are not differentiated according to the CP they apply to. This assumption is made for simplicity but is not crucial for our results. We will relax it in Section 5.1.

In our model, ISPs are so-called "access" ISPs that provide last mile connection to final consumers (Krämer, Wiewiorra and Weinhardt, 2013). We do not model the Internet backbone because our focus is on termination pricing. We assume that ISPs have transit and peering agreements that allow consumers to receive data packets from any $\mathrm{CP}$ over the

\footnotetext{
${ }^{9}$ Horizontal differentiation is a standard assumption in models of competition between ISPs (see, e.g., Economides and Tag, 2012; Choi, Jeon and Kim, forthcoming; Bourreau, Kourandi and Valletti, 2015). Canonical interpretations for this assumption are brand differentiation and heterogeneity in services bundled with Internet access. A further interpretation is uneven geographical coverage. Evidence of this can be found in several countries, in both landline and wireless broadband markets. For instance, a recent report by Britsh regulator Ofcom shows that all the main wireless operators cover $90 \%$ or more of the UK population with their $3 \mathrm{G}$ network, but coverage of the $4 \mathrm{G}$ network is much less uniform (http://www.ibtimes.co.uk/bestworst-uk-4g-3g-mobile-broadband-networks-revealed-1474662). Another example is the landline broadband market in the US. The main cable operators hold local monopolies for high-speed services in several regional markets. A report by the FCC shows that about $20 \%$ of homes in the US have access to a single broadband provider for a service of up to $4 \mathrm{Mbits} / \mathrm{s}$. The figure rises to $30 \%$ and $55 \%$ for speeds up to $10 \mathrm{Mbit} / \mathrm{s}$ and $25 \mathrm{Mbits} / \mathrm{s}$, respectively (http://www.washingtonpost.com/blogs/the-switch/wp/2014/09/04/fcc-chairmana-duopoly-dominates-basic-internet-service-in-america/).

${ }^{10}$ Termination fees are modeled as lump-sum. Modeling them as per-consumer or unit of data would not change the results.
} 
Internet, provided the ISP terminates such traffic.

Consumers. There is a mass (normalized to one) of consumers uniformly distributed on the $[0,1]$ interval. Consumers subscribe to one and only one ISP (the case of multi-homing consumers is considered in Section 5.3). The utility $U_{i}$ a consumer located in $x \in[0,1]$ gets from connecting to ISP $i$ is

$$
U_{i}(x)=Z+\Delta_{i}-t\left|x-l_{i}\right|-a_{i}, \quad i=A, B
$$

where $Z$ is the gross surplus from accessing the Internet, assumed large enough for all consumers to connect to an ISP. $t$ is the transportation cost and $l_{i}$ the ISP's position on the Hotelling line, so $l_{A}=0$ and $l_{B}=1$. Hence, $t\left|x-l_{i}\right|$ is the disutility for consumer $x$ from not subscribing to the ISP which is closest to her preferences. $\Delta_{i}$ represents the utility consumers get from browsing content available on ISP $i$. We assume that

$$
\Delta_{i}= \begin{cases}0 & \text { if no CP is available on ISP } i \\ \delta & \text { if one CP is available on ISP } i \\ \delta(1+\gamma) & \text { if both are available }\end{cases}
$$

with $\delta, \gamma \geq 0$. The term $\delta$ represents the utility consumers obtain from accessing a single content, while $\delta \gamma$ is the extra utility obtained by accessing an additional one. Hence, $\delta$ can be interpreted as the value consumers attribute to content availability in absolute terms. As for $\gamma$, it captures the extent to which utility changes with the quantity of available content. This may depend, for instance, on consumers' opportunity cost of time or the extent to which their attention span is limited. ${ }^{11}$ Furthermore, it may depend on consumers' preference for variety. Lastly, $\gamma$ may capture the degree of complementarity between contents. For example, two general interest news sites could be substitute, while a hotel booking website (e.g. Booking.com) and a maps website (e.g. Google Maps) could be complementary. One would then expect $\gamma$ to be larger in the latter case than in the former.

We do not endogenize consumers' demand for content: we simply assume that each consumer visits each available $\mathrm{CP}$ a certain number of times, normalized (without loss of generality) to one. We ignore (dis)utility from advertisements. ${ }^{12}$ We further assume that the

\footnotetext{
${ }^{11}$ If incremental utility is non-increasing with content availability, one should assume $\gamma \leq 1$. This would be of no consequence for the results that follow.

${ }^{12}$ This could be introduced with no significant change in the analysis. In our model, the quantity of impressions per content visited is invariant with the network configuration. Thus, nuisance caused by advertisements can simply be incorporated in the same way as $\delta$ and $\gamma$. These can therefore be interpreted as the incremental utility of content availability net of ad nuisance costs.
} 
market is covered and that demand for each ISP $i$ is positive, i.e. $\sum_{i} q_{i}=1$ and $q_{i}>0$.

Net consumer surplus $(C S)$ can be computed as

$$
\begin{gathered}
C S=\int_{0}^{q_{A}}\left(Z+\Delta_{A}-t x-a_{A}\right) d x+\int_{q_{A}}^{1}\left(Z+\Delta_{B}-t(1-x)-a_{B}\right) d x= \\
Z+\sum_{i=A, B}\left(\Delta_{i}-a_{i}\right) q_{i}+\frac{t}{2}-t q_{B}-t q_{A}^{2} .
\end{gathered}
$$

Content Providers. Each CP $j=0,1$ provides free content to consumers, but charges advertisers. An advertiser pays a per impression price $p_{j}$ if and only if a consumer is exposed to its ad while browsing content $j$. There is no cost of providing ad impressions and CPs set a uniform price for all ads. We assume CPs do not discriminate consumers according to which ISP they access content from. We also assume there is no across-outlet tracking. Hence, a CP has no knowledge of whether a given consumer has already been impressed by a given ad while browsing the rival's content or not. This means that prices $p_{j}$ cannot be discriminated accordingly.

CPs are endowed with a rather stylized advertising technology. Each visit to a content produces one impression of all ads placed on it. It follows that the CP can sell the one impression per viewer to each advertiser at the most. This is obviously a simplification, but, as we will point out below, it is of little consequence for our results. The total volume of impressions of an ad put on $\mathrm{CP} j$ is equal to $q_{j}$, which is the quantity of consumers subscribing to the $\operatorname{ISP}(\mathrm{s})$ distributing $j$ 's content, i.e.

$$
q_{j}=\sum_{i=A, B} q_{i} I_{i j}, \quad j=0,1 \quad i=A, B
$$

The profit of CP $j$ is

$$
\pi_{j}=d_{j} p_{j} q_{j}-\sum_{i=A, B} F_{i j} I_{i j}, \quad j=0,1 \quad i=A, B
$$

where $d_{j}$ is the quantity of advertisers on $\mathrm{CP} j$.

Of course, one could consider the presence of more CPs. However, this would not overturn our qualitative findings. We will come back to this point in Section 4.3.2 (footnote 18) below.

Advertisers. There is a mass of size one of advertisers. Their willingness to pay for impressions crucially depends on the quantity of outlets through which they can reach consumers. Let $\bar{V}$ denote the gross advertiser surplus (e.g., the resulting increase in the expected value of 
sales) produced by a single impression on a consumer. Let $\overline{\bar{V}}$ be the gross surplus produced by an additional impression (of the same ad on the same consumer). Recent literature on online advertising suggests that ads seen multiple times are partially wasteful (see, e.g., Calvano and Jullien, 2012), because they reach already informed consumers and may therefore be squandered together with their attention. Athey, Calvano and Gans (2013) estimate that more than two thirds of ads in large campaigns are wasted, hitting the same receivers more than 10 times. Accordingly, we will assume throughout the analysis that

$$
\bar{V}>\overline{\bar{V}}>0
$$

This assumption has very important implications for our results, as will be shown below.

Advertisers can buy impressions from no, one or both CPs. We assume that, if an advertiser is indifferent about placing an ad on a CP, it will do so. Assuming an advertiser multi-homes (i.e. places an ad on both content providers), its total surplus is

$$
\bar{V} \bigcup_{j=0,1} q_{j}+\overline{\bar{V}} \bigcap_{j=0,1} q_{j}-\sum_{j=0,1} p_{j} q_{j}
$$

where $\bigcup_{j=0,1} q_{j}$ denotes the quantity of consumers accessing at least one content and $\bigcap_{j=0,1} q_{j}$ the quantity of consumers accessing both contents. If all advertisers multi-home, each consumer accessing at least a $\mathrm{CP}\left(\bigcup_{j=0,1} q_{j}\right)$ is impressed at least once by each ad. Consumers that access both $\operatorname{CPs}\left(\bigcap_{j=0,1} q_{j}\right)$ are impressed twice. Assuming instead that an advertiser single-homes, buying an impression only from $\mathrm{CP} j$, then only consumers who have access to $j$ are impressed. Thus, the advertiser's total surplus is

$$
\left(\bar{V}-p_{j}\right) q_{j}, \quad j=0,1
$$

Obviously, the surplus is zero if no ad impression is bought. Given the network configuration and prices $p_{j}$, the advertiser chooses the option that guarantees the highest surplus.

Total advertiser surplus is

$$
A S=\sum_{j=0,1} d_{s j}\left(\bar{V}-p_{j}\right) q_{j}+d_{m}\left(\bar{V} \bigcup_{j=0,1} q_{j}+\overline{\bar{V}} \bigcap_{j=0,1} q_{j}-\sum_{j=0,1} p_{j} q_{j}\right)
$$

where $d_{s j}$ is the quantity of advertisers that single-home on $\mathrm{CP} j$ and $d_{m}$ is the quantity of advertisers that multi-home.

The assumption that each visit to a CP leads to one impression per ad is not crucial for our results. We could assume that each visit to a CP leads to $M>1$ impressions of each ad 
with no change in the results: in that case, one would have to redefine $\bar{V}$ as the total value of the first $M$ impressions of the ad on a consumer, $\overline{\bar{V}}$ as the value of the next $M$ impressions and $p_{j}$ as the price charged for the $M$ impressions guaranteed by CP $j$.

Welfare. Social welfare $S W$ is the sum of consumer surplus, advertiser surplus and total profits:

$$
S W=C S+A S+\sum_{i=A, B} \pi_{i}+\sum_{j=0,1} \pi_{j} .
$$

It is easily seen that $S W$ coincides with the gross surplus generated by connections at the two ends of the market, i.e. with the gross surplus of consumers and advertisers. Indeed, payments collected by ISPs and CPs are simply transfers from other players and cancel out in the $S W$ formula. Replacing $C S, A S, \sum_{i=A, B} \pi_{i}$ and $\sum_{j=0,1} \pi_{j}$, we get

$$
S W=Z+\sum_{i=A, B} \Delta_{i} q_{i}+\frac{t}{2}-t q_{B}-t q_{A}^{2}+\sum_{j=0,1} d_{s j} \bar{V} q_{j}+d_{m}\left(\bar{V} \bigcup_{j=0,1} q_{j}+\overline{\bar{V}} \bigcap_{j=0,1} q_{j}\right) .
$$

It can easily be shown that $S W$ is maximized when Universal Connection is the network configuration, each ISP serves half of the consumer base and advertisers place ads on all CPs. Thus, in our framework, departing from Universal Connection is never socially desirable.

Timing and definition of equilibrium. The timing of the game is as follows: ${ }^{13}$

1. In the Unregulated Regime, ISP $A$ and $B$ simultaneously set termination fees $F_{i}$. Having observed $F_{i}$, CPs simultaneously decide which ISP they connect to (if any). In the Net Neutrality regime, termination fees are set to zero by rule.

2. ISP $A$ and $B$ simultaneously set subscription fees $a_{i}$. CPs simultaneously set (per impression) prices $p_{j}$. Advertisers buy impressions from CPs.

3. Consumers connect to their preferred ISP, visit the available CPs and get exposed to advertisements.

A Subgame-Perfect Nash Equilibrium (SPNE) of the game $\mathbf{E}=(\mathbf{F}, \mathbf{I}, \mathbf{p}, \mathbf{a}, \mathbf{q}, \mathbf{d})$ is a profile of termination fees $\mathbf{F}=\left(F_{A}, F_{B}\right)$, connection decisions $\mathbf{I}=\left(I_{A 0}, I_{A 1}, I_{B 0}, I_{B 1}\right)$, per impression prices $\mathbf{p}=\left(p_{0}, p_{1}\right)$, subscription fees $\mathbf{a}=\left(a_{A}, a_{B}\right)$ and demands $\mathbf{q}=\left(q_{A}, q_{B}\right), \mathbf{d}=\left(d_{0}, d_{1}\right)$ such that, at each stage, no player is better off deviating unilaterally. We will focus on pure-strategy SPNEs.

\footnotetext{
${ }^{13}$ We discuss alternative assumptions on the timing in Section 5.5 below.
} 
Our model is such that, for some couples $\left(F_{A}, F_{B}\right)$, CPs may coordinate on more than one network configuration. Hence, we have to make an assumption regarding the actions of CPs off the equilibrium path. Specifically, if a deviation by an ISP results in a couple $\left(F_{A}, F_{B}\right)$ such that several network configurations may take place, we need to adopt a criterion to univocally establish which one would emerge. This is necessary in order to univocally determine the payoff that ISPs can obtain from deviating, and hence characterize the SPNE of the game. We make the following assumption: consider an equilibrium candidate $\mathbf{E}^{\mathbf{C}}$ which includes a vector of fees $\mathbf{F}^{\mathbf{C}}=\left(F_{A}^{C}, F_{B}^{C}\right)$ and connection decisions $\mathbf{I}^{C}$. We assume that if, say, ISP $A$ deviates to $F_{A}^{d}$ and $\left(F_{A}^{d}, F_{B}^{C}\right)$ is such that CPs may coordinate on several network configurations, they coordinate on the one that brings the smallest profits for the deviator. ${ }^{14}$

\section{Solving the model}

We now turn to the solution of the model, proceeding by backward induction.

\subsection{Stage 3}

At stage 3, consumers choose which ISP they connect to, given the CPs available at each ISP $i$ and subscription fees $a_{i}$. We first determine the marginal consumer $\bar{x}$ who is indifferent about the two ISPs. Equating $U_{A}(x)=U_{B}(x)$ and solving for $x$ we find

$$
\bar{x}=\frac{1}{2}+\frac{\left(\Delta_{A}-\Delta_{B}\right)-\left(a_{A}-a_{B}\right)}{2 t} .
$$

Demand for ISP $A$ is given by all consumers to the left of $\bar{x}$ on the Hotelling line, while that for ISP $B$ is given by all consumers to its right. That is, $q_{A}=\bar{x}$ and $q_{B}=1-\bar{x}$.

\subsection{Stage 2}

At stage 2, ISPs simultaneously set subscription fees, given termination fees $F_{i}$ as well as CPs' connection decisions (set at stage 1) and anticipating consumers' behavior (at stage 3). Equilibrium fees are obtained as the solution to the system of first order conditions

\footnotetext{
${ }^{14}$ The equilibria we describe below are therefore such that, if several network configurations can emerge as a result of a deviation, a "bad expectation" configuration forms against the deviator (this is similar to, e.g., Caillaud and Jullien, 2003). Observe that this imposes no restriction on the set of SPNEs of our game: if a SPNE exists under any other criterion to resolve the multiplicity, it must also be part of the set of SPNEs we characterize. This is because we resolve the eventual multiplicity always "against" the deviator.
} 
$\frac{\partial \pi_{i}}{\partial a_{i}}=0, i=A, B$. The result is

$$
a_{i}^{*}=t+\frac{\left(\Delta_{i}-\Delta_{i^{\prime}}\right)}{3}, \quad i=A, B \quad i^{\prime} \neq i
$$

Replacing $a_{i}^{*}$ in $q_{i}$ and $\pi_{i}$, we get (subgame) equilibrium demands and profits ${ }^{15}$

$$
q_{i}^{*}=\frac{1}{2}+\frac{\left(\Delta_{i}-\Delta_{i^{\prime}}\right)}{6 t}, \quad \pi_{i}^{*}=\frac{\left(3 t+\Delta_{i}-\Delta_{i^{\prime}}\right)^{2}}{18 t}+\sum_{j=0,1} I_{i j} F_{i}, \quad i=A, B \quad i^{\prime} \neq i
$$

Profits of platform $i$ are increasing in $\Delta_{i}-\Delta_{i^{\prime}}$ : a uniform increase in content availability for both ISPs leaves profits unchanged.

In order to describe the equilibrium demands conditional on $\Delta_{i}-\Delta_{i^{\prime}}$ briefly, we introduce some additional notation, summarized in Table 1.

\begin{tabular}{|c|c|c|c|c|c|}
\hline$\Delta_{i}-\Delta_{i^{\prime}}$ & $q_{i}^{*}$ & Denoted as & $\Delta_{i}-\Delta_{i^{\prime}}$ & $q_{i}^{*}$ & Denoted as \\
\hline 0 & $\frac{1}{2}$ & & 0 & $\frac{1}{2}$ & \\
\hline$-\delta$ & $\frac{1}{2}-\frac{\delta}{6 t}$ & $q^{--}$ & $\delta$ & $\frac{1}{2}+\frac{\delta}{6 t}$ & $q^{++}$ \\
$-\delta \gamma$ & $\frac{1}{2}-\frac{\delta \gamma}{6 t}$ & $q^{-}$ & $\delta \gamma$ & $\frac{1}{2}+\frac{\delta \gamma}{6 t}$ & $q^{+}$ \\
\hline$\delta(1+\gamma)$ & $\frac{1}{2}-\frac{\delta(1+\gamma)}{6 t}$ & $q^{---}$ & $\delta(1+\gamma)$ & $\frac{1}{2}+\frac{\delta(1+\gamma)}{6 t}$ & $q^{+++}$ \\
\hline
\end{tabular}

Table 1: Notation for consumer demands.

At this stage of the game, CPs also set the prices of ad impressions. We now informally describe how equilibrium prices are formed (see the Appendix for a formal derivation). Assume, to begin, that Universal Connection is the network configuration. The equilibrium is such that CPs charge a per impression price $p_{j}^{*}=\overline{\bar{V}}, j=0,1$ and advertisers multi-home. Competition for advertisers drives the price down to $\overline{\bar{V}} \cdot{ }^{16}$ A higher price could be profitably undercut by the rival CP, winning it the entire market. This is because advertisers would single-home on the cheapest outlet, still reaching all available consumers. On the other hand,

\footnotetext{
${ }^{15}$ Recall that we assume $\sum q_{i}^{*}=1$ and $q_{i}^{*}>0 \forall i$. This implies that $\delta(1+\gamma)<3 t$ holds.

${ }^{16}$ This is what Anderson, Foros and Kind (2014) define the "principle of incremental pricing".
} 
charging less than $\overline{\bar{V}}$ is clearly suboptimal: advertisers would still multi-home, because the price would be below the value of an additional ad impression $\overline{\bar{V}}{ }^{17}$

Assume now that Total Fragmentation is the network configuration. Each CP is the unique gatekeeper to the attention of subscribers to a ISP: it can therefore set a price $p_{j}^{*}=\bar{V}, j=0,1$, capturing the entire advertiser surplus. Finally, consider the Partial Fragmentation configuration: the CP that connects to both ISPs is the only available one just for some of the consumers (the other consumers are reached also by the other CP). The per impression price it charges turns out to be a weighted average of $\bar{V}$ and $\overline{\bar{V}}$, with weights given by the share of consumers reached once and twice, respectively. The other CP (that connects only to a single ISP but has to compete with the rival content to attract advertisers) charges instead a price equal to $\overline{\bar{V}}$.

The above discussion introduces Lemma 1, where we provide a complete description of profits made by CPs, conditionally on connection status.

Lemma 1 Profits $\pi_{j}^{*}$ of $C P j=0,1$, conditional on the network configuration, are as reported in Table 2.

Before proceeding, it is worthwhile emphasizing the importance of the advertising market and of overlapping audiences in our framework. Because repeated impressions are partially wasteful, an advertiser's willingness to pay for an impression diminishes when audiences of different CPs overlap. Therefore, CPs may obtain more revenue by serving a small exclusive audience rather than a large one that can browse other content. Indeed, when consumers can browse only one content, the $\mathrm{CP}$ is the unique gatekeeper to their attention. As a result, it is able to command a premium from advertisers (see, e.g., Athey, Calvano and Gans, 2013). This premium is particularly large when the surplus generated by a first ad impression $(\bar{V})$ is large compared to that of a repeated one $(\overline{\bar{V}})$. As we argue below, this mechanism is at the heart of the forces driving network fragmentation.

\subsection{Stage 1}

Let us now consider stage 1. We begin from the benchmark case of Net Neutrality and consider the Unregulated Regime next.

\footnotetext{
${ }^{17}$ If multiple impressions did not lose value, competition would have no impact on prices. On the other hand, if multi-homing by advertisers were ruled out, the equilibrium price would be equal to the marginal cost of impressions (which is zero by assumption).
} 


\begin{tabular}{|c|c|c|c|c|}
\hline $\begin{array}{l}0 \\
0 \\
z \\
Z\end{array}$ & $\begin{array}{c}0 \\
\dot{\tilde{i}} \\
\dot{\omega} \\
\dot{j} \\
\dot{1} \\
\dot{1}\end{array}$ & 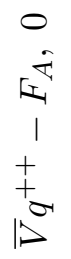 & $\begin{array}{l}0 \\
0 \\
x^{\infty} \\
1 \\
+ \\
+ \\
1^{\circ}\end{array}$ & $\begin{array}{l}0 \\
0\end{array}$ \\
\hline$\infty$ & 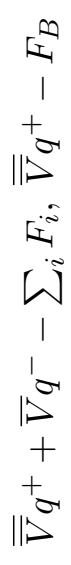 & 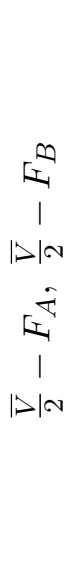 & 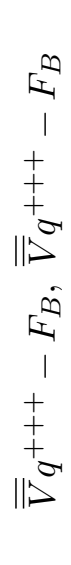 & $\begin{array}{l}x^{\infty} \\
1 \\
+ \\
+ \\
1^{+} \\
0\end{array}$ \\
\hline$\nabla$ & 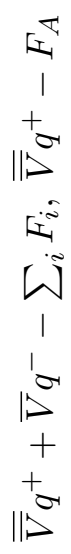 & 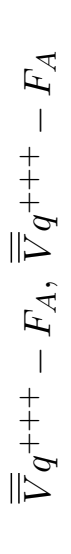 & i & $\begin{array}{l}x^{\top} \\
1 \\
+ \\
+ \\
1^{+} \\
0 \\
0\end{array}$ \\
\hline$\stackrel{\infty}{2}$ & 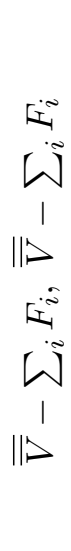 & 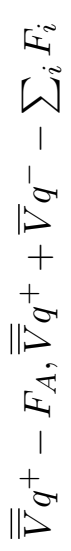 & 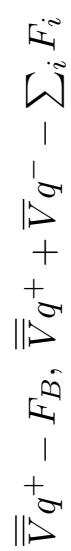 & $\underset{\mid}{\mid \vec{x}}$ \\
\hline $\begin{array}{l}\text { ठ } \\
\stackrel{8}{0}\end{array}$ & $\frac{2}{2}$ & $\nabla$ & $\infty$ & 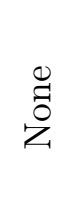 \\
\hline
\end{tabular}




\subsubsection{Benchmark: Net Neutrality}

In the Net Neutrality regime, ISPs have to terminate all traffic free of charge, so $F_{i}=0, i=$ $A, B$. As a result, any $\mathrm{CP}$ can have its content delivered to any consumer for free. Recall that we assume ISPs have transit agreements that allow consumers to receive data packets from any $\mathrm{CP}$ over the Internet, provided the ISP terminates such traffic. Moreover, we rule out the possibility that CPs unilaterally deny access to their content to specific consumers, based on the ISP they subscribe to. Consequently, in the Net Neutrality regime any content is available to all consumers the moment it goes online: Universal Connection is the unique equilibrium configuration. ${ }^{18}$ This provides us with a clear benchmark for comparison with the Unregulated Regime.

\subsubsection{Unregulated Regime}

Let us now turn to the Unregulated Regime. At stage 1, ISPs set termination fees $F_{i}, i=$ $A, B$ and CPs choose to which ISP they connect (i.e. want their traffic terminated from). Therefore, in contrast to the Net Neutrality regime, consumers subscribing to ISP $i$ have access to a content only as long as the $\mathrm{CP}$ agrees to pay $F_{i}$. The main question is whether (and under which conditions) this may alter the network configuration with respect to the Net Neutrality regime. Proposition 1 provides the answer.

\section{Proposition 1 In the Unregulated Regime:}

- The equilibrium network configuration is Total Fragmentation if and only if $\overline{\bar{V}}<S_{T F}$;

- The equilibrium network configuration is Universal Connection if and only if $S_{U C} \leq \overline{\bar{V}}$;

- No equilibrium entailing Partial Fragmentation exists;

where $S_{U C} \equiv \bar{V}\left(\frac{3 t}{6 t+\delta}\right)>S_{T F} \equiv\left(\frac{\bar{V}}{2}-\frac{\delta(1+\gamma)(6 t+\delta(1+\gamma))}{18 t}\right) \frac{3 t}{3 t+\delta(1+\gamma)}$.

Proposition 1 contains our basic result: termination fees may produce a fragmented Internet. This, we find, can take place when competition for advertisers substantially reduces

\footnotetext{
${ }^{18}$ Assuming that CPs do not discriminate among consumers connected to different ISPs seems to be a reasonable description of the current state of affairs. Moreover, this assumption allows us to focus squarely on the pricing decisions of ISPs, which are central to the Net Neutrality debate. Theoretically, though, one may perhaps envisage the possibility that, even if Net Neutrality were enforced, CPs exclude consumers depending on which ISPs they subscribe to. Nonetheless, allowing this would not fundamentally change our results. It can be shown that, while fragmentation could arise under Net Neutrality for some parameter values, Universal Connection would always be an equilibrium. Furthermore, it would be unique under a weaker condition than that we present below for the Unregulated Regime (see Proposition 1). In addition, equilibria involving fragmentation with Net Neutrality would not be robust to alternative assumptions concerning the timing of the game. We will return to this point in Section 5.5 below.
} 
ad rates (i.e. $\overline{\bar{V}}$ is low with respect to $\bar{V}$ ) and/or consumer demand is not strongly affected by total content value (i.e. $\gamma$ and $\delta$ are relatively small compared to $t$ ). In this equilibrium, each ISP sets a high termination fee: $F_{i}=\frac{\bar{V}}{2}, i=A, B$. Because the fee is equal to the profit made by selling exclusive ad impressions (on the attainable audience, of size $\frac{1}{2}$ ), the CP is willing to pay only if it does not have to face competition for the ISP's consumer base. As a result, each CP connects to a different ISP. ${ }^{19}$ The most profitable deviation for an ISP (say, $A$ ) is to reduce its fee to $\overline{\bar{V}} q^{+++}$, that is, to the point that it attracts both CPs exclusively. By so doing, ISP $A$ becomes more attractive for consumers than the rival. However, when $\overline{\bar{V}}$ is low with respect to $\bar{V}$ and/or $\gamma$ and $\delta$ are relatively small compared to $t$, the cost of this deviation on the content side of the market (lower revenues from termination fees) is larger than the gain on the consumer side (higher revenues from subscriptions fees). The intuition is simple. As we have seen above, overlapping audiences lead to repeated impressions, putting downward pressure on their price. When the price reduction is large, advertising revenues get a strong boost from exclusive viewership. Because they can recover advertising profits via termination fees, ISPs behave as editors, caring about the profitability of the content they carry. Hence, we have Total Fragmentation if and only if extra revenues from termination fees outweigh the additional consumer subscriptions an ISP obtains when offering more content than the competitor.

Proposition 1 states, in addition, that although Universal Connection is no longer guaranteed in the Unregulated Regime, it is not ruled out entirely. Suppose that competition among CPs has a relatively small impact on ad rates (i.e. $\overline{\bar{V}}$ is high enough with respect to $\bar{V})$ and/or that consumer demand is highly affected by content value (i.e. $\delta$ is sufficiently large compared to $t$ ). When such conditions hold, ISPs coordinate on an equilibrium where Universal Connection is preserved: content availability to consumers is maximized and all CPs are charged a relatively low fee (as we show in Appendix, $F_{i} \leq \overline{\bar{V}} q^{-}, i=A, B$ when Universal Connection is the equilibrium). The most profitable deviation from this equilibrium for an ISP is to raise its termination fee to the point that it induces Total Fragmentation. ${ }^{20}$

\footnotetext{
${ }^{19} \mathrm{By}$ a similar reasoning, one may suspect that there may exist an equilibrium where one $\mathrm{CP}$ is excluded from the market and the other is carried by both ISPs. However, this is never the case. Suppose ISP $A$ carries only CP 0 . ISP $B$ is always better off carrying exclusively CP 1 rather than carrying only 0 as well. The reason is that $\mathrm{CP} 1$ is willing to pay a higher termination fee as its outside option is weaker than that of $\mathrm{CP} 0$.

${ }^{20}$ It might be surprising to some extent that the deviation induces Total, not Partial, Fragmentation. The informal explanation is as follows: suppose an ISP (say, $A$ ), deviates by raising its fee to drop one CP (say, 0 ). Then CP 1 enjoys exclusive access to consumers of $A$, but as long as $\gamma>0$ some of them switch to $B$, because it can now offer more content. Given that the value of exclusive consumers is relatively large (otherwise $A$ would not deviate in the first place), CP 1 finds it profitable to abandon $B$. As a result, the quantity of exclusive eyeballs in 1's audience increases (due to the fact that both ISPs would offer only one content as a result). In the case where $\gamma=0$, the deviation inducing Partial Fragmentation brings to $A$ exactly the same profits as that inducing Total Fragmentation. See the Proof of Proposition 1 for a formal
} 
By so doing, the ISP gives up revenues from one $\mathrm{CP}$, but extracts more revenues from the remaining one (which enjoys exclusive access to the ISP's consumers). However, when the conditions mentioned above hold, such extra revenues extracted from the $\mathrm{CP}$ are insufficient to make the deviation profitable. ${ }^{21}$

Proposition 1 also asserts that there is no equilibrium entailing Partial Fragmentation. To see why, consider the following example. Suppose CP 0 connects only to ISP $A$ and CP 1 to both $A$ and $B$. In this configuration, CP 1 enjoys exclusive access to subscribers of ISP $B$. Yet, the tariff that $B$ is able to charge is limited in two instances. First, when the value of exclusive viewership on the advertising market is low (in particular, if $\overline{\bar{V}}$ is relatively close to $\bar{V})$. Second, when by offering one more content, $A$ is much more attractive as a platform for consumers, thereby providing advertisers with a much larger subscriber base. When either of the above is relevant, ISP $B$ is better off deviating to a fee that attracts both CPs, inducing Universal Connection. On the other hand, when none of the above is relevant, $A$ is better off "dropping" one of the CPs it carries, inducing Total Fragmentation. As we show in the Proof, it is never the case that both deviations are unprofitable. If the value of exclusive eyeballs is such that $B$ grants access to one $\mathrm{CP}$ only, then $A$ is better off doing the same. By contrast, when exclusive eyeballs sell at a low premium and $A$ retains both $\mathrm{CPs}, B$ is also better off getting them both.

Finally, we note that $S_{U C}$ is larger than $S_{T F}$. This implies that no pure-strategy equilibrium exists for some values of the parameters. ${ }^{22}$ The next paragraph provides further discussion on how thresholds $S_{U C}$ and $S_{T F}$ vary with the model's main parameters.

To summarize, Proposition 1 shows that there is an important relation between termination fees, network configuration and the online advertising market. In sum, the mechanism by which the profitability of online content decreases with the degree of audience overlapping is an important driver of fragmentation (should a zero price rule not be implemented). ${ }^{23}$ The welfare implications are rather straightforward. Only ISPs benefit from abandoning the Net

argument.

${ }^{21}$ Revenues from the consumer side are equal to $\frac{t}{2}$ under both Universal Connection and Total Fragmentation because ISPs offer access to an equal number of CPs.

${ }^{22}$ Of course, one could look for mixed-strategy equilibria. We do not engage in this exercise for two reasons. First, finding such equilibria is quite cumbersome. This is because at stage 1 each ISP has a continuous strategic variable (the termination fee) while the payoff function is non-continuous (given that network configurations vary non-continuously with fees). Second, our main result (that abandoning Net Neutrality may entail network fragmentation) would not be affected.

${ }^{23}$ The reader may wonder how our analysis would change if more than two CPs (identical to 0 and 1 ) were considered. While this would make the model less tractable, the basic mechanism we described above would not change. As long as audiences overlap, CPs would still have to compete for advertisers. Moreover, the more CPs consumers visit, the greater the chance they will be exposed to an ad several times. Thus, the link between termination and fragmentation would persist. 
Neutrality regime, while CPs are worse off. ${ }^{24}$ It is also easy to verify that neither consumer nor total welfare increase in the Unregulated Regime. We nonetheless stop short of formulating any normative prescription, as our model is not well-suited for an exhaustive welfare analysis. However, it points to the risk of fragmentation when termination fees are allowed, providing an argument in favor of adopting Net Neutrality regulation.

Comparative statics. We here describe in greater detail how the key parameters in consumer utility affect the network configuration. Consider $\delta$ and $\gamma$. $S_{T F}$ decreases in both. This means that the larger these parameters, the more stringent the condition for Total Fragmentation. The reason for this is that the most profitable deviation for an ISP, say, $A$, is to cut the termination fee to attract both CPs exclusively. The advantage of this move for $A$ is that its offer becomes more attractive to consumers than $B$ 's, to an extent that positively depends on $\delta$ and $\gamma$.

$S_{U C}$ is decreasing only in $\delta$. Hence, the condition for Universal Connection gets less stringent the larger $\delta$ is, but is independent of $\gamma$. The intuition is not entirely straightforward. There are two effects of these parameters. First, as we show in the Proof of Proposition 1, the most profitable deviation for an ISP from Universal Connection induces Total Fragmentation. Suppose ISP $A$ deviates raising its fee, dropping CP 0. ISP $B$ 's fee is relatively low (i.e. not greater than $\overline{\bar{V}} q^{-}$), which makes the option of connecting only to $B$ viable for $\mathrm{CP} 1$ and more attractive the larger $\delta$ and $\gamma$ are. Thus, the upper bound on the fee charged by $A$ when deviating decreases with both parameters. Second, ISP's profits with Universal Connection are decreasing in $\delta$ and $\gamma$, because $\delta$ and $\gamma$ drive down the fee that ISPs can charge if they want to keep all CPs on board. Indeed, should a CP abandon one ISP, it would make the other ISP more attractive to consumers, and the magnitude of this effect increases with $\delta$ and $\gamma$. Nevertheless, concerning $\delta$, the first effect prevails and, thus, greater content attractiveness slackens the condition for Universal Connection. As for $\gamma$, instead, it turns out that the two effects exactly cancel each other out.

We provide a graphical illustration of the effect of $\delta$ and $\gamma$ on thresholds $S_{T F}$ and $S_{U C}$ in Figure 2. The above findings are summarized in the following Proposition:

\section{Proposition 2 In the Unregulated Regime:}

- the more consumers value content in absolute terms (i.e. the larger $\delta$ is), the more (resp.

\footnotetext{
${ }^{24}$ Under Net Neutrality, ISP $i=A, B$ obtains $\pi_{i}^{U}=\frac{t}{2}$, while in the Unregulated Regime it obtains $\pi_{i}^{U}=\frac{t}{2}+2 \overline{\bar{V}} q^{-}$with Universal Connection and $\pi_{i}^{U}=\frac{t}{2}+\frac{\bar{V}}{2}$ with Total Fragmentation. CP $j=0,1$ makes $\pi_{j}^{N}=\overline{\bar{V}}$ under Net Neutrality, while it earns $\pi_{j}^{U}=\overline{\bar{V}}-2 \overline{\bar{V}} q^{-}$with Universal Connection and $\pi_{j}^{U}=0$ with Total Fragmentation in the Unregulated Regime.
} 


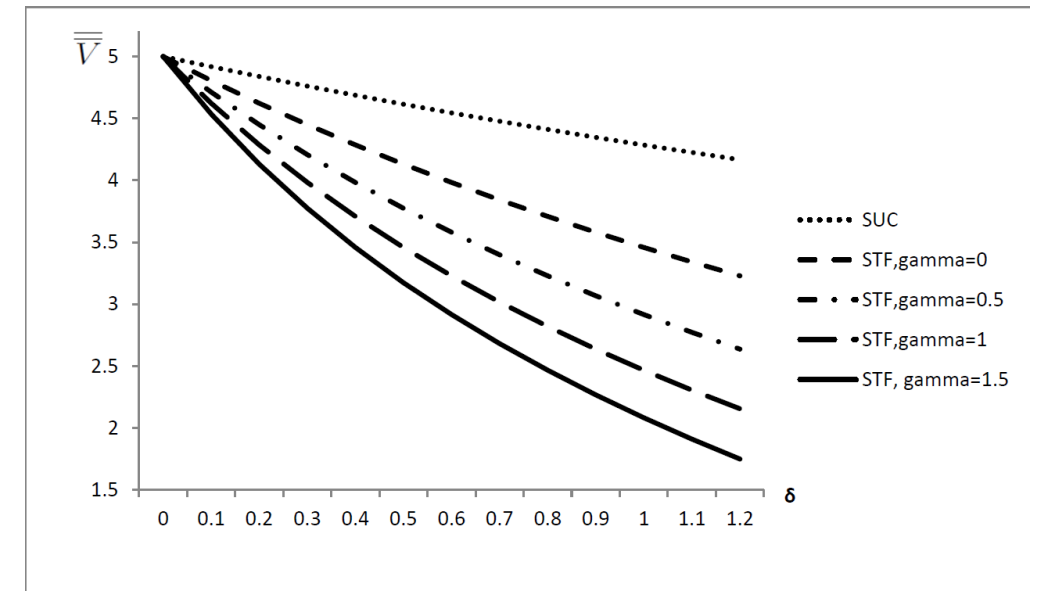

Figure 2: Illustration of how thresholds $S_{T F}$ and $S_{U C}$ vary with $\delta$ and $\gamma$. In this example $t=1, \bar{V}=10$

less) restrictive the condition for Total Fragmentation (resp. Universal Connection);

- the more contents are substitutable (i.e. the lower $\gamma$ is), the less restrictive the condition for Total Fragmentation.

Proof. See the discussion above.

Proposition 2 suggests that, all else given, Total Fragmentation is less likely to occur when contents are highly valuable and complementary for consumers. Furthermore, Universal Connection is more likely to emerge when consumers place high value on content. From a policy perspective, these findings are worth noting. To a certain extent, they weaken concerns about lifiting Net Neutrality restrictions. Nevertheless, one has to keep in mind that the advertising market is also crucial in determining the configuration. According to our results, fragmentation may emerge even if consumers strongly value content availability, as long as exclusive eyeballs can be sold at a large premium to advertisers.

\section{Extensions}

In this Section, we present several extensions to the baseline model. We will begin by considering discriminatory termination fees. Then, we will consider the distinction between mainstream and small CPs, alternative contractual arrangements for the sale of ad impressions (i.e. outsourcing to advertising platforms either run by third parties or by ISPs), multihoming consumers, vertical integration of CPs and ISPs. Finally, we will discuss alternative timings for our model. 


\subsection{Discriminatory termination fees}

The baseline model of Section 4 assumes that each ISP charges all CPs the same termination fee. We referred to this assumption as "uniform fees". We now assume that ISPs can charge different termination fees according to which $\mathrm{CP}$ they apply to: that is, $i$ sets a termination fee $F_{i j}$ that $\mathrm{CP} j$ has to pay in order to reach $i$ 's consumers. We will refer to this alternative assumption as "discriminatory fees".

The analysis of stages 3 and 2 of the model is unchanged with respect to Section 4 . However, the solution to stage 1 is not the same: by increasing the set of strategies available to ISPs, discriminatory fees expand the set of equilibria and deviations that have to be evaluated. Yet, the main results of Proposition 1 are robust to this change of scenario, as claimed in the following Proposition:

Proposition 3 With discriminatory fees in the Unregulated Regime, the sufficient condition for Total Fragmentation is weaker than $\overline{\bar{V}}<S_{T F}$. By contrast, the necessary and sufficient condition for Universal Connection, $\overline{\bar{V}} \geq S_{U C}$, is unchanged.

As the Proposition shows, discriminatory fees do not change (and in fact strengthen) our basic results. ${ }^{25}$ The intuition goes as follows. Uniform fees imply that, in the Total Fragmentation equilibrium, all ISPs charge a high fee to both CPs (and only one of them agrees to pay). Discriminatory fees allow greater flexibility. Indeed, we show in the Proof that a Total Fragmentation equilibrium exists such that each ISP charges a high fee (equal to $\frac{\bar{V}}{2}$ ) only to the $\mathrm{CP}$ it terminates traffic of. The other $\mathrm{CP}$ is instead charged a lower fee (though still large enough to be rejected). ${ }^{26}$ As a result, if an ISP deviated to attract both $\mathrm{CPs}$, it would have to match the low fee set by the rival. This makes the deviation less profitable than when fees are uniform, thereby expanding the region of parameters for which Total Fragmentation occurs. ${ }^{27}$

On the other hand, the uniform fees restriction is not relevant for Universal Connection.

\footnotetext{
${ }^{25}$ We show in the Supplementary Appendix that Partial Fragmentation is never an equilibrium.

${ }^{26}$ Formally, $F_{A 0}=F_{B 1}=\frac{\bar{V}}{2}, F_{A 1}=F_{B 0}<\frac{\bar{V}}{2}$ and CP 0 (resp. 1) connects to ISP $A$ (resp. B) only. The necessary and sufficient conditon for existence of this equilibrium is weaker than $\overline{\bar{V}}<S_{T F}$. Note that the equilibrium is not Pareto-optimal from the point of view of CPs: they could both be better off by switching connections. Nonetheless, the above set of fees would not be a mutual best response for the ISPs. In the Proof we also show that, as in the baseline model, an equilibrium entailing Total Fragmentation such that $F_{i j}=\frac{\bar{V}}{2}, i=A, B j=0,1$ exists if and only if $\overline{\bar{V}}<S_{T F}$ holds.

${ }^{27}$ With discriminatory termination fees, for some parameter values, both an equilibrium entailing Total Fragmentation and one entailing Universal Connection exist. This happens because the condition for Total Fragmentation is weaker than in the baseline model. However, because a full characterization of Total Fragmentation equilibria is not necessary to establish the results of Proposition 3, we will not describe conditions under which multiplicity occurs.
} 
In a nutshell, the reason is that when an ISP deviates by dropping a CP, it only has to worry about one of them accepting its offer.

To sum up, discriminatory fees make it easier for ISPs to coordinate on Total Fragmentation and have no effect on Universal Connection. Hence, relaxing the uniform fees restriction can only strengthen the basic conclusions of Proposition 1.

\subsubsection{Mainstream and small CPs.}

Proposition 3 suggests that discriminatory fees might facilitate Total Fragmentation. Hence, one may argue, on the condition that a zero-price rule is not adopted, a regulator may want to impose uniformity of termination fees. However, this choice may have some drawbacks. We have so far assumed that all CPs are equally valuable to consumers and advertisers. In reality, though, the Internet ecosystem consists of relatively few mainstream CPs coexisting with a plethora of smaller ones (e.g. blogs, forums). Because mainstream CPs capture most of consumers' attention (and advertising revenues), a reasonable concern is that abandoning Net Neutrality may not only produce fragmentation, but also exclusion of smaller content. Therefore, the presence of small CPs has implications on the degree of flexibility that a regulator may allow in setting termination fees. For the sake of brevity, we will here only discuss the issue informally. However, to support our arguments, we provide a stylized model in the Supplementary Appendix, where two mainstream CPs (i.e. 0 and 1 of the baseline model) coexist with a continuum of small CPs.

Suppose termination fees are allowed and consider the following two alternatives: 1) a full discrimination regime, where each ISP can charge each mainstream CP a different fee, as well as charging small CPs a separate fee; 2) in a uniform fees regime, each ISP has to charge all CPs the same fee, regardless of their nature. As the results of Proposition 3 suggest, with full discrimination, ISPs can more easily coordinate on Total Fragmentation than when fees have to be uniform. On the other hand, given that they can charge a different fee to small CPs, they have little reason to exclude them. On the contrary, if ISPs cannot discriminate,

they may choose to price small CPs out of their network in order to extract more profits from mainstream ones. In sum, this suggests a trade-off between protecting accessibility of mainstream content and plurality of secondary content.

\section{$5.2 \quad$ Foreclosure and outsourcing of ad impressions}

As we noted in Section 2, our model can be interpreted as a theory of vertical foreclosure (Rey and Tirole, 2007). Each ISP controls access to its subscribers as a monopolist. CPs then sell consumer eyeballs to advertisers. A CP can access all consumers connected to an 
ISP by paying the termination fee. Due to the decreasing marginal value of impressing a consumer with an ad, competition among CPs drives down the price of impressions. Thus, an ISP may want to foreclose a $\mathrm{CP}$, fragmenting the network, in order to protect the remaining one from competition. The latter is then able to obtain monopolistic (downstream) profits, which the ISP can recoup through the termination fee.

It is important to note that, in our baseline framework, foreclosure (and, hence, fragmentation) may occur because ISPs are otherwise unable to extend their market power downstream. In reality, though, ISPs and CPs might enter into contractual arrangements different to the ones considered so far. Specifically, we have assumed that CPs manage ad impressions internally. However, it is conceivable that they might outsource management of impressions to third-party platforms (e.g. those run by Google or Yahoo). A further possibility is that ISPs set up their own advertising platforms. It is therefore interesting to investigate how our results may be affected if impressions were sold according to such alternative arrangements. As the results below show, foreclosure is of no use if a single third-party platform controls all the advertising market (though not if CPs outsource impressions to different advertising platforms). Foreclosure is also unnecessary if the ISP can directly control the price and quantity of ad impressions on its consumers.

Third-party ad platforms. Let us begin with the scenario in which advertising is outsourced to third-party platforms, e.g. Google. Suppose first there is only one such platform, acting as a monopolist. We assume that the advertising platform controls the price $p_{j}, j=0,1$ of an impression on each content and transfers to the respective $\mathrm{CP}$ a share $\alpha \in[0,1]$ of the per impression revenue (for simplicity, we assume that this share is exogenous). As we show in Appendix, because pricing is fully in the hands of the advertising platform, the profitability of impressions is independent of the degree of audience overlapping. Intuitively, this eliminates the driving force behind fragmentation.

However, the results of Proposition 1 are still valid if there are two advertising platforms, each serving one CP: competition among them again reduces advertising profits in presence of overlapping viewership. It follows that fragmentation can take place should termination fees be introduced, as in the baseline model. The only caveat is that CPs have to retain a non-negligible share of advertising revenues (i.e. $\alpha$ is large enough). Otherwise, their ability to pay termination fees is too low and, hence, ISPs never induce fragmentation.

Proposition 4. If CPs outsource advertising to a monopolist third-party platform, Universal Connection is the unique equilibrium configuration regardless of the regulatory regime. However, if each CP outsources to a different third-party platform, Total Fragmentation can 
still emerge in the Unregulated Regime as long as CPs retain a share of ad revenues $\alpha$ that is not too small.

The Proposition suggests an interesting link between the market power of third-party advertising platforms and Internet fragmentation in presence of termination fees: while competition among platforms obviously reduces market power, it may also increase the risk of fragmentation. Thus, if termination fees were allowed, the presence of a dominant advertising platform may, indirectly, have a beneficial effect on content availability.

ISP-run ad platforms. We now consider a different scenario, in which management of advertising is outsourced to ISPs. ${ }^{28}$ It should be intuitive that if each ISP is the unique gatekeeper to the attention of its subscribers, irrespectively of which content they browse, overlapping viewership has no impact on prices of impressions. By the same token, all incentives for ISPs to fragment the network are eliminated.

Proposition 5. If CPs outsource advertising impressions to ISPs, Universal Connection is the unique equilibrium configuration regardless of the regulatory regime.

Allowing ISPs to run their own advertising platforms may thus reduce the risk of network fragmentation. A drawback is that such platforms could in principle exercise a dominant position on the advertising market. A perhaps even more relevant policy concern, which is outside the scope of our work, is that advertising technologies implemented at the ISP level are potentially able to track consumer behavior more intrusively than other platforms (see, e.g., European Commission, 2009).

\subsection{Multi-homing subscribers to ISPs}

We have hitherto assumed that consumers single-home when connecting to the Internet. Although this is currently a widespread practice, with a fragmented network consumers may wish to subscribe to multiple ISPs and access all content. This, one may conjecture, could defuse the threat of fragmentation. To shed some light on the issue, we will now relax the single-homing assumption. To avoid repetition, we describe only informally the changes to the baseline model and the main result. The formal analysis is available in the Supplementary Appendix.

\footnotetext{
${ }^{28}$ Some ISPs seem to have taken steps in this direction. Consider, for instance, the case of British ISPs BT, TalkTalk and VirginMedia who signed an agreement with technology vendor Phorm to launch the new ad platform, OIX, in 2008 (see http://www.theguardian.com/technology/2008/mar/06/internet.privacy).
} 


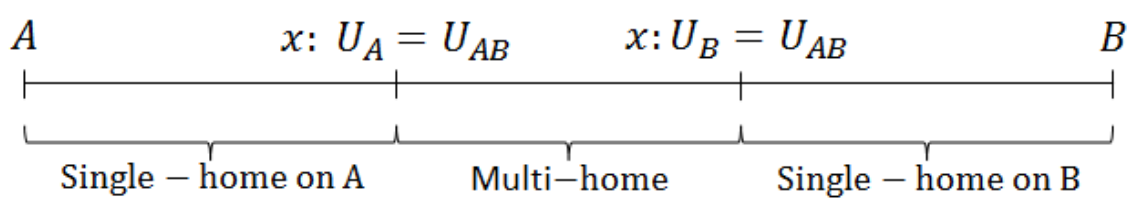

Figure 3: Consumer demands for ISPs with multi-homing.

Suppose consumers have the same utility function as in the baseline model, but they can now choose to subscribe to both ISPs. ${ }^{29}$ Assume also that consumers do not have any extrautility from accessing the same content through several ISPs. That is, the incremental utility from subscribing to an additional ISP is positive if and only if this allows to access content unavailable otherwise. Consequently, consumers multi-home only if the network configuration is Total Fragmentation. ${ }^{30}$ Moreover, only consumers located (sufficiently) close to the middle of the Hotelling line multi-home in equilibrium. Intuitively, the quantity of multi-homers increases with the utility from visiting an additional content. It decreases instead in the transportation cost $t$, which makes joining both platforms more costly. ${ }^{31}$ Figure 3 provides a graphical representation of consumer demands when Total Fragmentation is the network configuration.

Let us now describe how multi-homing affects prices of ad impressions. Because they visit all content, multi-homers are impressed twice by each ad. Hence, their eyeballs cannot be sold at the monopoly price by CPs, despite fragmentation. As a result, with Total Fragmentation the equilibrium price of impressions is smaller than in the baseline model (i.e. below $\bar{V}$ ). Furthermore, it is decreasing in the quantity of multi-homers. In any other configuration impressions have the same price as in the baseline model because all consumers single-home.

We are now ready to state the main result: Total Fragmentation is possible despite multihoming. Suppose the quantity of multi-homers is relatively low (i.e. the attractiveness of additional content is relatively small) and repeated impressions have relatively little value. CPs are still willing to pay a high price to be the only CP terminated by an ISP because they can provide some valuable exclusive consumers to advertisers. As a result, Total Fragmentation still emerges in equilibrium. Hence, our baseline result is, on the whole, robust to the presence of multi-homing consumers. However, it can be shown that Total Fragmentation

\footnotetext{
${ }^{29}$ Our formal model of consumer multi-homing follows Choi (2010).

${ }^{30}$ Multi-homing is a dominated alternative for consumers when all other network configurations occur. This is obvious when Universal Connection is the network configuration. As for Partial Fragmentation, subscribing only to the ISP that carries both contents strictly dominates multi-homing (due to the transportation cost).

${ }^{31}$ The quantity of single-homers therefore increases when ISPs are more differentiated. See footnote 9 above for a discussion of the assumption of horizontal differentiation of ISPs.
} 
occurs only within a subset of the region of parameters for which it occurs in the baseline model. The simple intuition is that, as explained above, multi-homers weaken the mechanism whereby fragmentation creates exclusive audiences and inflates impression prices.

\subsection{Vertical integration of CPs and ISPs with Net Neutrality}

Our results so far suggest that Net Neutrality, and a ban on termination fees in particular, prevent ISPs from fragmenting the network. Yet, one could argue that ISPs could react to such regulation by vertically integrating with CPs. This may put them in a position to restrain availability of proprietary content to subscribers of the rival platform. ${ }^{32}$ Net Neutrality might therefore not be enough to protect universal content availability.

In order to investigate the above issues, we here consider the Net Neutrality regime in presence of vertically integrated firms. Our basic model is adapted in the following way: we add a preliminary stage (stage 0), where ISPs simultaneously decide whether to integrate with a CP. We assume that, despite Net Neutrality regulation, an integrated ISP has the option of making proprietary content available only to its own subscribers. ${ }^{33}$ In stage 1 , integrated ISPs (if any) decide simultaneously whether to make such content available on the rival platform. This determines the network configuration.

For brevity, we will here illustrate the main results. We refer the reader to the Supplementary Appendix for a formal proof. It is useful to begin by taking the outcome of stage 0 as given. Suppose ISP $B$ is integrated with CP 1 and ISP $A$ with CP 0. Proceeding backwards, stage 3 remains as in the baseline model. At stage 2, integrated ISPs simultaneously choose the subscription fee and the price of ad impressions. We show in the Supplementary Appendix that, depending on whether the proprietary content is made available to the rival ISP, equilibrium profits of the integrated firms are as reported in Table 3.

\begin{tabular}{|c|c|c|}
\hline$C P 0+I S P A$ & Offer content to rival & Do not offer \\
\hline \hline Offer content to rival & $\overline{\bar{V}}+\frac{t}{2}, \overline{\bar{V}}+\frac{t}{2}$ & $\overline{\bar{V}}+\frac{(3 t-\delta \gamma+\bar{V}-2 \overline{\bar{V}})^{2}}{18 t}, \frac{(3 t+\delta \gamma-\bar{V}+2 \overline{\bar{V}})^{2}}{18 t}$ \\
\hline Do not offer & $\frac{(3 t+\delta \gamma-\bar{V}+2 \overline{\bar{V}})^{2}}{18 t}, \overline{\bar{V}}+\frac{(3 t-\delta \gamma+\bar{V}-2 \overline{\bar{V}})^{2}}{18 t}$ & $\frac{t}{2}, \frac{t}{2}$ \\
\hline
\end{tabular}

Table 3: Profits of the vertically integrated firms " $B+1$ " and " $A+0$ " in the Net Neutrality regime, conditionally on whether the proprietary content is offered to the rival firm or not.

\footnotetext{
${ }^{32}$ Concerns of this kind were expressed by the FCC, with reference to the recent merger of Comcast and NBC-Universal (Waterman and Choi, 2011).

${ }^{33}$ ISPs may make exclusive content accessible only to their subscribers, e.g. via proprietary modems or smartphone apps.
} 
Consider now stage 1. Table 3 suggests that, unlike in our basic model with separated firms, Total Fragmentation is possible despite Net Neutrality. Both ISPs decide not to offer their proprietary content to the rival if and only if $\frac{t}{2}>\overline{\bar{V}}+\frac{(3 t-\delta \gamma+\bar{V}-2 \overline{\bar{V}})^{2}}{18 t}$. The latter holds as long as $t$ and $\delta \gamma$ are large and/or, most interestingly, the value of both first and second impressions is relatively small. This stands in contrast to the baseline model (see Proposition 1). The intuition is that integrated firms face a trade-off when deciding whether to make proprietary content universally available. On the one hand, exclusive content raises the value of the ISP to consumers and provides a monopolistic advantage when selling ad impressions. On the other hand, exclusivity limits the proprietary content's audience. Moreover, integrated ISPs internalize cross-group externalities between consumers and advertisers. Hence, the greater the profitability of ad impressions, the greater the incentive to subsidize consumers. ${ }^{34}$ Indeed, the gross surplus from selling exclusive ad impressions $\frac{\bar{V}}{2}$ is entirely transferred to consumers and equilibrium profits are only $\frac{t}{2}$. Table 3 also suggests that Universal Connection occurs if and only if $\overline{\bar{V}}+\frac{t}{2} \geq \frac{(3 t+\delta \gamma-\bar{V}+2 \overline{\bar{V}})^{2}}{18 t}$. This condition is more likely to hold when $\delta \gamma$ is small and when the profitability of selling exclusive eyeballs to advertisers (captured by $\bar{V})$ is large. The intuition reflects the one provided for Total Fragmentation.

A quick comparison to the baseline model suggests that, when both ISPs are vertically integrated, Total Fragmentation can emerge even when $\overline{\bar{V}} \geq S_{U C}$ holds. In other words, we can have Total Fragmentation even if Universal Connection would be the equilibrium with separated firms (in the Unregulated Regime). ${ }^{35}$

Suppose now that only one ISP is integrated with a CP. We find that this ISP does not necessarily deviate from Universal Connection. Yet, Universal Connection takes place under weaker conditions when both ISPs vertically integrate. This depends on the fact that a nonintegrated ISP is more accommodating when setting subscription fees as it does not take into account cross-market externalities (i.e. between advertisers and consumers). Hence, the option not to provide the integrated content is more attractive when the rival ISP is independent.

Let us now take a step back to stage 0 . The question is whether and when ISPs actually have incentives to integrate vertically. While a complete answer cannot be easily provided, we find that when one ISP chooses not to merge with a CP, the other ISP always finds it profitable to do so. Hence, at least one integrated platform always forms (see the Appendix for the proof).

\footnotetext{
${ }^{34}$ See, e.g, Armstrong (2006), Caillaud and Jullien (2003), Rochet and Tirole (2006).

${ }^{35} \mathrm{~A}$ complete comparison of conditions for which Fragmentation and Universal Connection are equilibria with vertical integration and in the baseline model turned out to be rather cumbersome. This is due to the fact that profits in Table 3 are quadratic functions of $\bar{V}$ and $\overline{\bar{V}}$.
} 
To summarize, Net Neutrality does not necessarily prevent fragmentation in presence of vertically integrated ISPs. A regulator might therefore want to intervene imposing must-offer requirements to integrated firms. ${ }^{36}$

We observe, on the other hand, that while profits made by integrated ISP-CPs with Universal Connection are the same as in the baseline model (i.e. $\overline{\bar{V}}+\frac{t}{2}$ ), profits with Total Fragmentation are strictly smaller than if CPs and ISPs are separated (all else equal). We speculate therefore that vertical mergers are less appealing for a ISP-CP couple, should termination fees be allowed. This applies, in particular, when conditions leading to Total Fragmentation with vertically separated firms (as outlined in Proposition 1) hold.

\subsection{The role of timing in our model}

We now discuss our assumptions regarding the timing of the game. We have assumed that CPs make connection decisions before consumers subscribe to ISPs. This affects the way in which the latter decide on fees. In a standard two-sided market framework, where ISPS simultaneously decide subscription and termination fees, it may occur that ISPs cut subscription prices in order to attract more consumers and exploit cross-group externalities. The timing we assume rules this out, because competition for consumers comes at a later stage with respect to competition for content (as in, e.g., Choi, Jeon and Kim, forthcoming, and Kourandi, Krämer and Valletti, 2015). However, we believe this to be a reasonable simplification, justified by the fact that in general contracts between ISPs and CPs have a more long-run perspective and their connection arrangements are more rigid than those between ISPs and consumers.

Nonetheless, there may be reasons to consider a different timing, in which competition for consumers comes before competition for content. On the one hand, consumer demand for ISPs may react rather slowly to changes in content availability, for instance because of switching costs. On the other, if they were able and willing to do so, CPs might quickly block consumers of a given ISP from accessing their content. ${ }^{37}$ It is therefore useful to consider how our results would be affected by adopting a different timing in which consumers subscribe to ISPs before CPs make connection decisions. ${ }^{38}$ First, it is easy to conclude that Universal

\footnotetext{
${ }^{36}$ In the TV market, must-offer obligation has been at times imposed as a remedy for vertical mergers. For instance, the European Commission imposed to Newscorp-Telepiù to offer proprietary content on the wholesale market. A similar requirement was imposed by British regulator Ofcom on BSkyB in relation to its control over sports channels.

${ }^{37}$ Recall that, in our baseline model, CPs are not able to discriminate among consumers connected to different ISPs.

${ }^{38}$ Specifically, the game would unfold as follows. First, ISPs set subscription fees and consumers choose their ISP. Second, ISPs set termination fees (if applicable) and CPs choose which ISP to connect to. Third, ad prices are set and impressions bought by advertisers.
} 
Connection would be the unique equilibrium configuration with Net Neutrality. Even if it were able to unilaterally exclude consumers of an ISP from its content, no CP would have any incentive to do so. With given consumer demands, it would always be a dominant strategy for any CP to offer its content to all consumers. Turning now to the Unregulated Regime, ISPs anticipate the effect of changing the consumer base on connection decisions of CPs (and on termination fees) at the final stage. Hence, the problem ISPs solve when setting the subscription fee would become non-continuous and, thus, significantly more complex than in our basic model. Despite this, we can reasonably expect that Total Fragmentation would still be an equilibrium in the Unregulated Regime, as long as $\bar{V}$ is sufficiently large compared to $\overline{\bar{V}}$ (note that the pricing of ad impressions would not change with respect to the current model). Thus, we conjecture that the main insights of our investigation would be robust to this change of timing.

\section{Concluding remarks}

We have investigated the relation between Net Neutrality, Internet fragmentation and the advertising market. We have shown that when competition among CPs has a relevant impact on advertising rates, letting ISPs charge termination fees may induce fragmentation of the Internet. CPs may prefer not to ask for traffic termination if faced with fees that are too high. Complaints of exclusion by CPs might call for a price cap on termination fees. However, computing the adequate cap is likely to be a difficult task for the regulator, which reinforces the argument in favor of a zero-price rule. Nevertheless, our results also suggest that termination fees do not systematically lead to fragmentation. When competition among CPs has little impact on advertising rates, Universal Connection emerges in equilibrium regardless of the regime. Moreover, Net Neutrality may not guarantee a universally connected network if ISPs and CPs are integrated.

Before concluding, we would like to discuss some aspects of the Net Neutrality debate that have been left out of our analysis. First of all, we have ignored network congestion, which could be alleviated by service tiering and priority fees. However, we believe modeling congestion would not change our findings concerning fragmentation. In the Unregulated Regime, ISPs could grant priority to some traffic while reducing quality of service for the rest. It is therefore conceivable that CPs that do not acquire priority from a given ISP would become much less attractive for consumers, losing appeal as advertising outlets. On the contrary, those that acquire priority could command much greater ad rates than their non-prioritized rivals. Therefore, CPs may find it rational to acquire priority at different ISPs, leading de facto to a fragmented Internet. 
A second central aspect we have ignored are incentives to invest in infrastructure and content provision. These may of course be affected by the regulatory regime. While the study of such issues is beyond the scope of our paper, it seems reasonable to think that the main forces we have highlighted would be relevant even if investment were endogenized. 


\section{Appendix}

Notation. In order to concisely describe the network configurations, we now introduce some notation. In the following, $(0 A B, 1 A B)$ means that 0 connects to both $A$ and $B$ and 1 connects to both $A$ and $B$ (i.e. $I_{i j}=1 \forall i, j$ ): in the text, we refer to this configuration as "Universal Connection". $\left(0 i, 1 i^{\prime}\right)$ means that 0 connects only to $i$ and 1 connects only to $i^{\prime}$ (i.e. $I_{i 0}=I_{i^{\prime} 1}=1 I_{i 1}=I_{i^{\prime} 0}=0$ ): in the text, we refer to this configuration as "Total Fragmentation". $\left(j i, j^{\prime} A B\right)$ means that CP $j$ connects to ISP $i$ and CP $j^{\prime}$ connects to both (i.e. $I_{A j^{\prime}}=I_{B j^{\prime}}=1 I_{A j} \neq I_{B j}$ ): in the text, we refer to this configuration as "Partial Fragmentation". Finally, $\left(j N, j^{\prime} A B\right)$ means that CP $j$ connects to no ISP and CP $j^{\prime}$ connects to both $A$ and $B,\left(I_{A j^{\prime}}=I_{B j^{\prime}}=1 I_{A j}=I_{B j}=0\right)$. Similar notation is used for all other configurations.

\section{Proof of Lemma 1}

Making use of (2), (6) and Table 1, as well as advertiser surplus in (3) and (4), we will obtain impression prices and equilibrium profits of CPs in each network configuration.

Configuration $(0 A B, 1 A B)$. If an advertiser puts an ad on both CPs, it gets $\bar{V}+\overline{\bar{V}}-p_{0}-p_{1}$. If instead the ad is put only on $\mathrm{CP} i$, the advertiser gets $\bar{V}-p_{i}$. This implies that an advertiser multi-homes if and only if $\bar{V}+\overline{\bar{V}}-p_{0}-p_{1} \geq \bar{V}-p_{j}$, i.e. $\overline{\bar{V}} \geq p_{j}$. We now prove that $p_{j}=\overline{\bar{V}}, j=0,1$ are the equilibrium impressions prices and all advertisers multi-home. Consider the best response function of CP 1 . Suppose $p_{0} \leq \overline{\bar{V}}$ : when $p_{1} \leq \overline{\bar{V}}$, advertisers multi-home. If $p_{1}>\overline{\bar{V}}$ no advertiser puts an ad on CP 1 . It follows that $p_{1}=\overline{\bar{V}}$ is the best response for CP 1 . Suppose now $p_{0}>\overline{\bar{V}}$ : if $p_{1}>p_{0}$, all advertisers single-home on CP 0 . If $p_{1}<p_{0}$, all advertisers single-home on CP 1 . Finally, if $p_{1}=p_{0}$, each advertiser single-homes and is indifferent between the two CPs. We assume that in this case advertisers split equally between them. It follows that the best response for CP 1 is $p_{1}=p_{0}-\varepsilon$. Repeating the reasoning for CP 0 , it is easily seen that the only mutual best response is $p_{j}^{*}=\overline{\bar{V}}, j=0,1$. As a result, all advertisers multi-home and $\pi_{j}=\overline{\bar{V}}-F_{A}-F_{B}, j=0,1$.

Configuration $\left(j A B, j^{\prime} i\right), i=A, B, j, j^{\prime}=0,1$ and $j \neq j^{\prime}$. Consider the case $(0 A B, 1 A) .{ }^{39}$ If an advertiser multi-homes, it gets $\left(\bar{V}-p_{0}\right) q^{-}+\left(\bar{V}+\overline{\bar{V}}-p_{0}-p_{1}\right) q^{+}$, given that there are $q^{B}=q^{-}$exclusive consumers and $q^{A}=q^{+}$non-exclusive consumers. If an advertiser single-homes on CP 0 , it gets $\bar{V}-p_{0}$. Finally, if the advertiser single-homes on CP 1 , it gets $\left(\bar{V}-p_{1}\right) q^{+}$, because only $q^{+}$consumers are impressed (once) by its ad, while the remaining

\footnotetext{
${ }^{39}$ The proof is specular in all other configurations of this kind.
} 
consumers are not impressed at all. We will prove that advertisers multi-home and CPs set the following equilibrium prices: $p_{1}=\overline{\bar{V}}$ and $p_{0}=\bar{V} q^{-}+\overline{\bar{V}} q^{+}$.

Let us start by the best response function of CP 0. Suppose $p_{1} \leq \overline{\bar{V}}$. Advertisers always put ads on CP 1, irrespectively of whether they multi-home: $p_{1}$ is below the value of a repeated impression. For an advertiser already putting an ad on CP 1, however, multi-homing is profitable only if $\left(\bar{V}-p_{0}\right) q^{-}+\left(\bar{V}+\overline{\bar{V}}-p_{0}-p_{1}\right) q^{+} \geq\left(\bar{V}-p_{1}\right) q^{+}$, i.e. $p_{0} \leq \bar{V} q^{-}+\overline{\bar{V}} q^{+}$ holds. In that case, $\pi_{0}=p_{0}-F_{A}-F_{B}$. If instead $p_{0}>\bar{V} q^{-}+\overline{\bar{V}} q^{+}$, all advertisers single-home on CP 1 and $\pi_{0}=-F_{A}-F_{B}$. It follows that the best response for 0 is $p_{0}=\bar{V} q^{-}+\overline{\bar{V}} q^{+}$. Suppose now $p_{1}>\overline{\bar{V}}$ : an advertiser putting an ad on CP 0 does not multi-home. Hence, either all advertisers single-home on $\mathrm{CP} 0$ or on $\mathrm{CP} 1$. CP 0 is better off being the outlet chosen, which is the case if and only if $\left(\bar{V}-p_{0}\right) \geq\left(\bar{V}-p_{1}\right) q^{+}$, i.e. $p_{0} \leq \bar{V} q^{-}+p_{1} q^{+}$, holds. Thus, the best response for 0 is $p_{0}=\bar{V} q^{-}+p_{1} q^{+}$.

Let us now describe the best response function for CP 1 . Suppose $p_{0} \leq \bar{V} q^{-}+\overline{\bar{V}} q^{+}$. Advertisers multi-home if $p_{1} \leq \overline{\bar{V}}$ holds. If $p_{1}>\overline{\bar{V}}$ all advertisers single-home on CP 0 , because $\left(\bar{V}-p_{0}\right)>\left(\bar{V}-p_{1}\right) q^{+}$. Hence, the best response for $\mathrm{CP} 1$ is $p_{1}=\overline{\bar{V}}$. Suppose now that $p_{0}>\bar{V} q^{-}+\overline{\bar{V}} q^{+}$. Again, all advertisers multi-home if $p_{1} \leq \overline{\bar{V}}$ holds. If instead $p_{1}>\overline{\bar{V}}$, advertisers single-home on CP 0 if $\left(\bar{V}-p_{0}\right) \geq\left(\bar{V}-p_{1}\right) q^{+}$and single-home on CP 1 otherwise. Thus, if CP 1 sets $p_{1} \leq \overline{\bar{V}}, \pi_{1}=p_{1} q^{+}-F_{A}$. If it sets $\overline{\bar{V}}<p_{1} \leq \frac{p_{0}-\bar{V} q^{-}}{q^{+}}$, it captures all the advertising market and $\pi_{1}=p_{1} q^{+}-F_{A}$. CP 1's best response is thus $p_{1}=\frac{p_{0}-\bar{V} q^{-}}{q^{+}}$. Given the above, the mutual best response is $p_{1}^{*}=\overline{\bar{V}}$ and $p_{0}^{*}=\bar{V} q^{-}+\overline{\bar{V}} q^{+}$. As a result we get $\pi_{1}^{*}=\overline{\bar{V}} q^{+}-F_{A}, \pi_{0}^{*}=\bar{V} q^{-}+\overline{\bar{V}} q^{+}-\sum_{i} F_{i}$ and that advertisers multi-home.

Configuration $(\mathbf{0} i, \mathbf{1} i), i=A, B$. With a reasoning similar to that $(0 A B, 1 A B)$, it can be proved that the equilibrium is such that $p_{0}^{*}=p_{1}^{*}=\overline{\bar{V}}$ so that CPs' profits are $\pi_{0}^{*}=\overline{\bar{V}} q^{+++}-F_{i}$ $\pi_{1}^{*}=\overline{\bar{V}} q^{+++}-F_{i}$. Again, all advertisers multi-home.

Configuration $\left(0 i, 1 i^{\prime}\right), i, i^{\prime}=A, B$ and $i \neq i^{\prime}$. If an advertiser puts ads on both CPs, its total surplus is $\left(\bar{V}-p_{0}\right) \frac{1}{2}+\left(\bar{V}-p_{1}\right) \frac{1}{2}$. If instead ads are put only on CP $j$, the surplus is $\left(\bar{V}-p_{j}\right) \frac{1}{2}$. Thus, it can be shown that $p_{0}^{*}=p_{1}^{*}=\bar{V}, \pi_{0}^{*}=\frac{\bar{V}}{2}-F_{i}$ and $\pi_{1}^{*}=\frac{\bar{V}}{2}-F_{i^{\prime}}$. In equilibrium advertisers multi-home.

Configuration $\left(j A B, j^{\prime} N\right), j, j^{\prime}=0,1$ and $j \neq j^{\prime}$. Consider the case $(0 A B, 1 N)$. No advertiser puts an ad on CP 1 because it does not provide access to any consumer. If an advertiser puts ads on CP 0 , its total surplus is $\bar{V}-p_{0}$. Thus, the only equilibrium is such that $p_{0}^{*}=\bar{V}$. Hence, CPs' profits are $\pi_{1}^{*}=0$ and $\pi_{0}^{*}=\bar{V}-\sum_{i} F_{i}$. All advertisers place ads only on $\mathrm{CP}$. A specular reasoning applies to configuration $(0 N, 1 A B)$. 
Configuration $\left(j \mathbf{N}, j^{\prime} i\right), i=A, B, j, j^{\prime}=0,1$ and $j \neq j^{\prime}$. Consider $(0 A, 1 N)$. No advertiser puts an ad on $\mathrm{CP} 1$ because no consumer is reached. If an advertiser puts ads on CP 0 , its total surplus is $\left(\bar{V}-p_{0}\right) q^{++}$. Thus, $p_{0}^{*}=\bar{V}$. Hence, CPs' profits are $\pi_{1}^{*}=0$ and $\pi_{0}^{*}=\bar{V} q^{+}-F_{A}$. A specular reasoning applies to $(0 N, 1 A),(0 N, 1 B)$ and $(0 B, 1 N)$.

\section{Proof of Proposition 1}

We proceed in two steps. Step 1 describes necessary conditions that termination fees have to satisfy in order for each network configuration to take place in equilibrium. In Step 2, we characterize the SPNEs.

STEP 1. Start from CPs' profits as described in Table 2. For a given network configuration $\left(I_{A 0}, I_{A 1}, I_{B 0}, I_{B 1}\right)$ to arise, it must be the case that, conditionally on $F_{A}$ and $F_{B}$, and given the connection choice $I_{A j^{\prime}}, I_{B j^{\prime}}$ of the rival $j^{\prime}$, choosing $I_{A j}, I_{B j}$ is the best response for CP $j$ (with $j=0,1$, ). One can therefore back up the conditions that have to be satisfied by $F_{A}$ and $F_{B}$ in order for any network configuration to constitute a mutual best response. These are reported below. We provide in Figure 4 an illustration of such conditions ${ }^{40}$. Observe that they are not always mutually exclusive. That is, for some couples $F_{A}$ and $F_{B}$ there may be more than one configuration constituting a mutual best response for CPs.

1. $(0 A B, 1 A B)$

$$
F_{i} \leq \overline{\bar{V}} q^{-}, i=A, B
$$

2. $(0 A, 1 A B)$ or $(0 A B, 1 A)$.

$$
\overline{\bar{V}} q^{-}<F_{B} \leq \bar{V} q^{-}+\overline{\bar{V}} q^{+}-\overline{\bar{V}} q^{+++}, \quad F_{A} \leq \bar{V} q^{-}+\overline{\bar{V}} q^{+}-\frac{\bar{V}}{2}, \quad F_{B} \geq F_{A} .
$$

3. $(0 B, 1 A B)$ or $(0 A B, 1 B)$.

$$
\overline{\bar{V}} q^{-}<F_{A} \leq \bar{V} q^{-}+\overline{\bar{V}} q^{+}-\overline{\bar{V}} q^{+++}, \quad F_{B} \leq \bar{V} q^{-}+\overline{\bar{V}} q^{+}-\frac{\bar{V}}{2}, \quad F_{A}>F_{B} .
$$

4. $(0 N, 1 A B)$ or $(0 A B, 1 N)$.

$$
\overline{\bar{V}} q^{+}<F_{i} \leq \bar{V} q^{--}, i=A, B .
$$

\footnotetext{
${ }^{40}$ we omit configurations $(0 N, 1 N),(0 N, 1 i)$ and $(0 N, 1 A B)$ and symmetric, because they are irrelevant for our arguments below
} 
5. $(0 A, 1 A)$

$$
F_{A} \leq \overline{\bar{V}} q^{+++}, F_{B}>\bar{V} q^{-}+\overline{\bar{V}} q^{+}-\overline{\bar{V}} q^{+++}, F_{B}-F_{A}>\frac{\bar{V}}{2}-\overline{\bar{V}} q^{+++} .
$$

Replace subscript $A$ with subscript $B$ for configuration $(0 B, 1 B)$.

6. $(0 A, 1 B)$ or $(0 B, 1 A)$

$$
\bar{V} q^{-}+\overline{\bar{V}} q^{+}-\frac{\bar{V}}{2}<F_{i} \leq \frac{\bar{V}}{2}, i=A, B, \quad \overline{\bar{V}} q^{+++}-\frac{\bar{V}}{2} \leq F_{A}-F_{B} \leq \frac{\bar{V}}{2}-\overline{\bar{V}} q^{+++} .
$$

7. $(0 N, 1 A)$ or $(0 A, 1 N)$.

$$
\overline{\bar{V}} q^{+++}<F_{A} \leq \bar{V} q^{++}, \quad \frac{\bar{V}}{2}<F_{B}, \quad F_{B}>F_{A} .
$$

Replace subscript $A$ with subscript $B$ for $(0 N, 1 B)$ or $(0 B, 1 N)$.

8. $(0 N, 1 N)$

$$
F_{i}>\bar{V} q^{++}, \quad i=A, B
$$

STEP 2. We proceed as follows. First, for each network configuration we identify the set of candidates to equilibrium $\left\{\mathbf{F}^{\mathbf{C}}\right\}$. A "candidate" is a couple of fees $\mathbf{F}^{\mathbf{C}} \equiv\left(F_{A}^{C}, F_{B}^{C}\right)$ that satisfies the relevant necessary conditions among (7) - (14) described in Step 1 . Second, we rule out candidates in $\left\{\mathbf{F}^{\mathbf{C}}\right\}$ that trivially cannot constitute an equilibrium entailing the given configuration. Finally, we describe the most profitable unilateral deviations from the remaining candidates. By so doing, we identify the necessary and sufficient conditions for each candidate to constitute an equilibrium.

Note that the relevant deviation from a candidate $\mathbf{F}^{\mathbf{C}} \equiv\left(F_{A}^{C}, F_{B}^{C}\right)$ depends on parameter values. In particular, it depends on how small $\overline{\bar{V}}$ is relatively to $\bar{V}$. Accordingly, in order to streamline our arguments, throughout the remainder of the proof we will divide the support $(0, \bar{V})$ of $\overline{\bar{V}}$ in several sub intervals and analyze deviations in each of them.

\section{Market configuration $(0 A B, 1 A B)$}

The set of candidates $\left\{\mathbf{F}^{\mathbf{C}}\right\}_{U C}$ inducing $(0 A B, 1 A B)$ must satisfy conditions (7). An element of $\left\{\mathbf{F}^{\mathbf{C}}\right\}_{U C}$ is therefore such that $F_{i}^{C} \leq \overline{\bar{V}} q^{-}$and generates profits $\pi_{i}^{C}=2 F_{i}^{C}+\frac{t}{2}$ for $i=A, B$. 


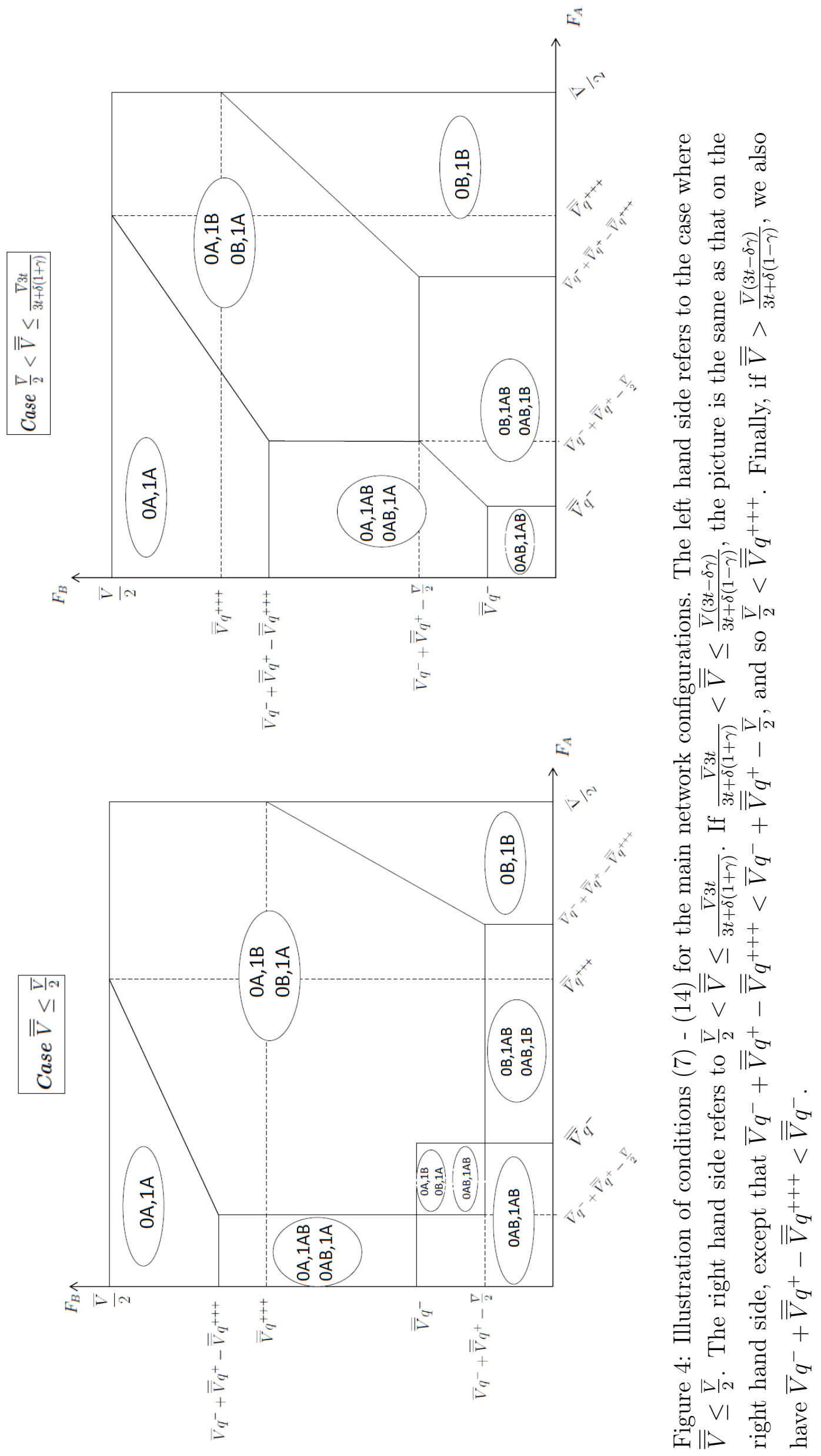


a. Case $\overline{\bar{V}} \leq \frac{\bar{V}}{2}$. Consider first the candidate such that $F_{i}^{C}=\overline{\bar{V}} q^{-}$and $\pi_{i}^{C}=2 \overline{\bar{V}} q^{-}+\frac{t}{2}$ for $i=A, B$. It is tempting to immediately rule out all the other candidates in $\left\{\mathbf{F}^{\mathbf{C}}\right\}_{U C}$, because an ISP could raise its fee to $F_{i}=\overline{\bar{V}} q^{-}$without violating (7). However, as we explain below, this deviation is not necessarily profitable due to a multiplicity issue. Nevertheless, it is useful to focus on this candidate first because, we will argue, any other candidate in $\left\{\mathbf{F}^{\mathbf{C}}\right\}_{U C}$ can constitute an equilibrium entailing $(0 A B, 1 A B)$ only if $F_{i}^{C}=\overline{\bar{V}} q^{-}$also does.

Suppose that ISP $A$ deviates. A deviation such that $F_{A}^{d}<F_{A}^{C}$ cannot be profitable, because $A$ would earn lower profits. If $F_{A}^{d}>F_{A}^{C}$, given that $F_{B}^{C}=\overline{\bar{V}} q^{-}$, the following configurations can be induced: ${ }^{41}$

$(0 B, 1 B)$ : because $F_{B}^{C}=\overline{\bar{V}} q^{-}<\overline{\bar{V}} q^{+++}$, this is induced if $A$ sets $F_{A}^{d}>\max \left\{\bar{V} q^{-}+\right.$ $\left.\overline{\bar{V}} q^{+}-\overline{\bar{V}} q^{+++} ; \frac{\bar{V}}{2}-\overline{\bar{V}} q^{+++}+\overline{\bar{V}} q^{-}\right\}$, so that only conditions (11) are satisfied. ISP $A$ earns $\pi_{A}^{d}=\frac{(3 t-\delta(1+\gamma))^{2}}{18 t}$.

- $\quad\left(j A, j^{\prime} B\right), j, j^{\prime}=0,1 j \neq j^{\prime}$ : ISP $A$ can induce this configuration in the most profitable way setting $F_{A}^{d}=\min \left\{\frac{\bar{V}}{2} ; \frac{\bar{V}}{2}-\overline{\bar{V}} q^{+++}+\overline{\bar{V}} q^{-}\right\}=\frac{\bar{V}}{2}-\overline{\bar{V}} q^{+++}+\overline{\bar{V}} q^{-}$, so that only conditions (12) are satisfied. ISP $A$ earns $\pi_{A}^{d}=\overline{\bar{V}} q^{-}+\frac{\bar{V}}{2}-\overline{\bar{V}} q^{+++}+\frac{t}{2}$.

Because $\frac{(3 t-\delta(1+\gamma))^{2}}{18 t}<\pi_{A}^{C}$, a deviation inducing $(0 B, 1 B)$ is not profitable. The deviation inducing $(0 A, 1 B)$ or $(0 B, 1 A)$ is profitable if and only if $\frac{\bar{V}}{2}-\overline{\bar{V}} q^{+++}>\overline{\bar{V}} q^{-}$. A specular reasoning applies to deviations by $B$. Hence, a necessary and sufficient condition for candidate $F_{i}^{C}=\overline{\bar{V}} q^{-}, i=A, B$ to constitute an equilibrium entailing $(0 A B, 1 A B)$, when $\overline{\bar{V}} \leq \frac{\bar{V}}{2}$ applies, is

$$
\frac{\bar{V}}{2}-\overline{\bar{V}} q^{+++} \leq \overline{\bar{V}} q^{-} \Leftrightarrow \overline{\bar{V}} \geq \bar{V}\left(\frac{3 t}{6 t+\delta}\right) \equiv S_{U C} .
$$

Let us now consider the remainder of $\left\{\mathbf{F}^{\mathbf{C}}\right\}_{U C}$. All candidates such that $F_{i}^{C} \leq \bar{V} q^{-}+$ $\overline{\bar{V}} q^{+}-\frac{\bar{V}}{2}$ for at least one $i=A, B$ cannot be equilibria. Because $\bar{V} q^{-}+\overline{\bar{V}} q^{+}-\frac{\bar{V}}{2}<\overline{\bar{V}} q^{-}$ holds when $\overline{\bar{V}} \leq \frac{\bar{V}}{2}$ applies, either one ISP could profitably deviate setting $F_{i}^{d}=\overline{\bar{V}} q^{-}$or one can deviate setting $F_{i}^{d}=\bar{V} q^{-}+\overline{\bar{V}} q^{+}-\frac{\bar{V}}{2}$, still inducing $(0 A B, 1 A B)$. It remains to consider candidates such that $\bar{V} q^{-}+\overline{\bar{V}} q^{+}-\frac{\bar{V}}{2}<F_{i}^{C}<F_{i}^{C}<\overline{\bar{V}} q^{-}$for $i=A, B$. If an ISP deviates setting $F_{A}^{d}=\overline{\bar{V}} q^{-}$, CPs can coordinate either on configurations $\left(j A, j^{\prime} B\right) j, j^{\prime}=0,1, j \neq j^{\prime}$ or $(0 A B, 1 A B)$. To solve this multiplicity issue, we invoke our "bad expectations" assumption (described extensively at page 11). Under this assumption, the former configuration is the

\footnotetext{
${ }^{41}$ Deviating to Partial Fragmentation is not feasible. Suppose ISP $A$ decided to deviate to $(0 B, 1 A B)$, "dropping" CP 0. Because, when Case a applies, $\bar{V} q^{-}+\overline{\bar{V}} q^{+}-\frac{\bar{V}}{2} \leq \overline{\bar{V}} q^{-}$holds, the necessary condition $F_{B} \leq \bar{V} q^{-}+\overline{\bar{V}} q^{+}-\frac{\bar{V}}{2}$ in $(9)$ is violated. Moreover, $A$ cannot deviate inducing $(0 A, 1 A B)$ or $(0 A B, 1 A)$ because $F_{B}^{C}=\overline{\bar{V}} q^{-}$, hence the necessary condition $\overline{\bar{V}} q^{-}<F_{B}$ in (8) is violated. Similar reasoning applies to $B$.
} 
relevant one. ISP $i$ can therefore most profitably deviate to $\left(j A, j^{\prime} B\right) j, j^{\prime}=0,1, j \neq j^{\prime}$, setting $F_{i}^{d}=F_{i^{\prime}}^{C}+\frac{\bar{V}}{2}-\overline{\bar{V}} q^{+++}, i \neq i^{\prime}$, obtaining $\pi_{i}^{d}=F_{i^{\prime}}^{C}+\frac{\bar{V}}{2}-\overline{\bar{V}} q^{+++}+\frac{t}{2} .{ }^{42}$ Using the same steps as above it can be shown that any such candidate constitutes an equilibrium entailing $(0 A B, 1 A B)$ if and only if $F_{i^{\prime}}^{C}+\frac{\bar{V}}{2}-\overline{\bar{V}} q^{+++} \leq 2 F_{i}^{C}$ for $i=A, B$ and $i^{\prime} \neq i$. It is easily seen that the latter conditions can jointly hold only if (15) does. Hence, an equilibrium entailing $(0 A B, 1 A B)$ exists, when $\overline{\bar{V}} \leq \frac{\bar{V}}{2}$ applies, if and only if (15) holds.

b. Case $\frac{\bar{V}}{2}<\overline{\bar{V}}$. Consider candidate $F_{i}^{C}=\overline{\bar{V}} q^{-}, i=A, B$. We study unilateral deviations by ISP $A$. A deviation such that $F_{A}^{d}<F_{A}^{C}$ cannot be profitable. Suppose ISP $A$ deviates by increasing $F_{A}$. The following configurations can be induced: ${ }^{43}$

$(0 B, 1 B)$ : because $F_{B}^{C}=\overline{\bar{V}} q^{-}<\overline{\bar{V}} q^{+++}$, this is induced if $A$ sets $F_{A}^{d}>\max \left\{\bar{V} q^{-+}\right.$ $\left.\overline{\bar{V}} q^{+}-\overline{\bar{V}} q^{+++} ; \frac{\bar{V}}{2}-\overline{\bar{V}} q^{+++}+\overline{\bar{V}} q^{-}\right\}$, so that only conditions (11) are satisfied. ISP $A$ earns $\pi_{i}^{d}=\frac{(3 t-\delta(1+\gamma))^{2}}{18 t}$.

$\left(j B, j^{\prime} A B\right) j, j^{\prime}=A, B, j^{\prime} \neq j$ : ISP $A$ can induce this by setting $F_{A}^{d}=\bar{V} q^{-}+$ $\overline{\bar{V}} q^{+}-\overline{\bar{V}} q^{+++}$, obtaining $\pi_{A}^{d}=\bar{V} q^{-}+\overline{\bar{V}} q^{+}-\overline{\bar{V}} q^{+++}+\frac{(3 t-\delta \gamma)^{2}}{18 t}$.

Because $\pi_{A}^{d}=\frac{(3 t-\delta(1+\gamma))^{2}}{18 t}<\pi_{A}^{C}$, a deviation inducing $(0 B, 1 B)$ is not profitable. A deviation inducing $(0 B, 1 A B)$ or $(0 A B, 1 B)$ is profitable if and only if $\bar{V} q^{-}+\overline{\bar{V}} q^{+}-\overline{\bar{V}} q^{+++}+\frac{(3 t-\delta \gamma)^{2}}{18 t}>$ $2 \overline{\bar{V}} q^{-}+\frac{t}{2}$. This does not hold, because $2 \overline{\bar{V}} q^{-}>\bar{V} q^{-}+\overline{\bar{V}} q^{+}-\overline{\bar{V}} q^{+++}$when $\frac{\bar{V}}{2}<\overline{\bar{V}}$ applies. We can follow a specular reasoning for deviations by $B$. Hence, an equilibrium entailing $(0 A B, 1 A B)$ always exists when $\frac{\bar{V}}{2}<\overline{\bar{V}}$ applies.

Summing up. We can conclude that Universal Connection is an equilibrium network configuration if and only if $\overline{\bar{V}} \geq S_{U C}$ holds. Any equilibrium entailing Universal Connection is such that $F_{i}^{C} \leq \overline{\bar{V}} q^{-}$for $i=A, B$.

\section{Market configuration $(0 A, 1 A B)$}

The set of candidates $\left\{\mathbf{F}^{\mathbf{C}}\right\}_{P F}$ that may induce this configuration has to satisfy (8). We focus on candidate $F_{A}^{C}=\bar{V} q^{-}+\overline{\bar{V}} q^{+}-\frac{\bar{V}}{2}, F_{B}^{C}=\bar{V} q^{-}+\overline{\bar{V}} q^{+}-\overline{\bar{V}} q^{+++}$. Any other candidate $\operatorname{in}\left\{\mathbf{F}^{\mathbf{C}}\right\}_{P F}$ is such that a least one ISP $i$ could profitably deviate by raising $F_{i}$ until the relevant constraint in (8) binds. ISP profits are $\pi_{A}^{C}=2\left(\bar{V} q^{-}+\overline{\bar{V}} q^{+}-\frac{\bar{V}}{2}\right)+\frac{(3 t+\delta \gamma)^{2}}{18 t}$ and $\pi_{B}^{C}=\bar{V} q^{-}+\overline{\bar{V}} q^{+}-\overline{\bar{V}} q^{+++}+\frac{(3 t-\delta \gamma)^{2}}{18 t}$.

\footnotetext{
${ }^{42}$ This issue does not arise when $F_{i}^{C} \leq \bar{V} q^{-}+\overline{\bar{V}} q^{+}-\frac{\bar{V}}{2}, i=A, B$, because conditions for $\left(0 i, 1 i^{\prime}\right) i=$ $A, B, i^{\prime} \neq i$ in (12) are violated.

${ }^{43}$ Configuration $\left(j A, j^{\prime} B\right) j, j^{\prime}=0,1, j \neq j^{\prime}$ cannot be induced by a unilateral deviation because $\overline{\bar{V}} q^{-}<$ $\bar{V} q^{-}+\overline{\bar{V}} q^{+}-\overline{\bar{V}} q^{+++}$holds when $\frac{\bar{V}}{2}<\overline{\bar{V}}$ applies.
} 
a. Case $\overline{\bar{V}} \leq \frac{\bar{V}}{2}$. First, consider unilateral deviations by $B$. Given that ISP $A$ sets $F_{A}^{C}=\bar{V} q^{-}+\overline{\bar{V}} q^{+}-\frac{\bar{V}}{2}, B$ can induce:

$(0 A B, 1 A B): B$ sets $F_{B}^{d}=\overline{\bar{V}} q^{-}$. This is such that only conditions (7) are satisfied. ${ }^{44}$ ISP $B$ obtains $\pi_{B}^{d}=2 \overline{\bar{V}} q^{-}+\frac{t}{2}$.

Consider now deviations by $A$. Given that $F_{B}^{C}=\bar{V} q^{-}+\overline{\bar{V}} q^{+}-\overline{\bar{V}} q^{+++}, A$ can induce:

$\left(j A, j^{\prime} B\right), j, j^{\prime}=A, B, j^{\prime} \neq j:$ ISP $A$ sets $F_{A}^{d}=\min \left\{\frac{\bar{V}}{2} ; \frac{\bar{V}}{2}-\overline{\bar{V}} q^{+++}+F_{B}^{C}\right\}=$ $\min \left\{\frac{\bar{V}}{2} ; \frac{\bar{V}}{2}+\bar{V} q^{-}+\overline{\bar{V}} q^{+}-2 \overline{\bar{V}} q^{+++}\right\}$. This is such that only conditions (12) are satisfied. ISP $B$ obtains $\pi_{A}^{d}=\min \left\{\frac{\bar{V}}{2} ; \frac{\bar{V}}{2}+\bar{V} q^{-}+\overline{\bar{V}} q^{+}-2 \overline{\bar{V}} q^{+++}\right\}+\frac{t}{2}$.

We will now prove that, when $\overline{\bar{V}} \leq \frac{\bar{V}}{2}$ applies, at least one among the following inequalities is satisfied:

$$
\begin{gathered}
\pi_{B}^{d}=2 \overline{\bar{V}} q^{-}+\frac{t}{2}>\pi_{B}^{C}, \\
\pi_{A}^{d}=\min \left\{\frac{\bar{V}}{2} ; \frac{\bar{V}}{2}+\bar{V} q^{-}+\overline{\bar{V}} q^{+}-2 \overline{\bar{V}} q^{+++}\right\}+\frac{t}{2}>\pi_{A}^{C} .
\end{gathered}
$$

This is sufficient to establish that a profitble deviation exists. Rearranging (16) we get

$$
\frac{\bar{V}}{2}-\overline{\bar{V}} q^{+++}<2 \overline{\bar{V}} q^{-}-\left(\bar{V} q^{-}+\overline{\bar{V}} q^{+}-\frac{\bar{V}}{2}\right)+\frac{t}{2}-\frac{(3 t-\gamma \delta)^{2}}{18 t}
$$

while rearranging (17) we get

$$
\begin{gathered}
\min \left\{\frac{\bar{V}}{2} ; \frac{\bar{V}}{2}+\bar{V} q^{-}+\overline{\bar{V}} q^{+}-2 \overline{\bar{V}} q^{+++}\right\}-\left(V^{\prime \prime} q^{+}-\bar{V}\left(\frac{1}{2}-q^{-}\right)\right)> \\
>\left(V^{\prime \prime} q^{+}-\bar{V}\left(\frac{1}{2}-q^{-}\right)\right)+\frac{(3 t+\delta \gamma)^{2}}{18 t}-\frac{t}{2} .
\end{gathered}
$$

Note that $\min \left\{\frac{\bar{V}}{2} ; \frac{\bar{V}}{2}+\bar{V} q^{-}+\overline{\bar{V}} q^{+}-2 \overline{\bar{V}} q^{+++}\right\}-\left(V^{\prime \prime} q^{+}-\bar{V}\left(\frac{1}{2}-q^{-}\right)\right)>\frac{\bar{V}}{2}-\overline{\bar{V}} q^{+++}$when $\overline{\bar{V}} \leq \frac{\bar{V}}{2}$ applies. Hence, a sufficient condition for at least one among (16) and (17) to hold is:

$$
2 \overline{\bar{V}} q^{-}-\left(\bar{V} q^{-}+\overline{\bar{V}} q^{+}-\frac{\bar{V}}{2}\right)+\frac{t}{2}-\frac{(3 t-\gamma \delta)^{2}}{18 t}>\left(\bar{V} q^{-}+\overline{\bar{V}} q^{+}-\frac{\bar{V}}{2}\right)+\frac{(3 t+\delta \gamma)^{2}}{18 t}-\frac{t}{2}
$$

\footnotetext{
${ }^{44}$ Note that, when Case a applies, $\overline{\bar{V}} q^{+}-V^{\prime}\left(\frac{1}{2}-q^{-}\right) \leq \overline{\bar{V}} q^{-}$holds.
} 
Because $\bar{V} q^{-}+\overline{\bar{V}} q^{+}-\frac{\bar{V}}{2} \leq \overline{\bar{V}} q^{-}$when $\overline{\bar{V}} \leq \frac{\bar{V}}{2}$ applies and because $\delta+\delta \gamma<3 t$ by assumption (see footnote 13 in the text), (18) is satisfied.

b. Case $\frac{\bar{V}}{2}<\overline{\bar{V}} \leq \frac{\bar{V}(3 t-\delta \gamma)}{3 t+\delta(1-\gamma)}$. Consider deviations by ISP $B$. Given that ISP $A$ sets $F_{A}^{C}=\bar{V} q^{-}+\overline{\bar{V}} q^{+}-\frac{\bar{V}}{2}, B$ can deviate inducing

$(0 B, 1 A B)$ or $(0 A B, 1 B):$ if $\bar{V} q^{-}+\overline{\bar{V}} q^{+}-\frac{\bar{V}}{2} \leq \bar{V} q^{-}+\overline{\bar{V}} q^{+}-\overline{\bar{V}} q^{+++}$, ISP $B$ sets $F_{B}^{d}=\min \left\{\bar{V} q^{-}+\overline{\bar{V}} q^{+}-\frac{\bar{V}}{2} ; F_{A}^{C}-\varepsilon\right\}$, with $\varepsilon$ small and positive, so only (9) are satisfied. In both cases $B$ obtains $\pi_{B}^{d}=2\left(\bar{V} q^{-}+\overline{\bar{V}} q^{+}-\frac{\bar{V}}{2}-\varepsilon\right)+\frac{(3 t+\delta \gamma)^{2}}{18 t}$.

$(0 B, 1 B):$ if $\bar{V} q^{-}+\overline{\bar{V}} q^{+}-\frac{\bar{V}}{2}>\bar{V} q^{-}+\overline{\bar{V}} q^{+}-\overline{\bar{V}} q^{+++}$, ISP $B$ sets $F_{B}^{d}=\bar{V} q^{-}+$ $\overline{\bar{V}} q^{+}-\overline{\bar{V}} q^{+++} \leq \min \left\{\overline{\bar{V}} q^{+++} ; F_{A}^{C}-\frac{\bar{V}}{2}+\overline{\bar{V}} q^{+++}-\varepsilon\right\}$, so only (11) are satisfied. $B$ obtains $\pi_{B}^{d}=2\left(\bar{V} q^{-}+\overline{\bar{V}} q^{+}-\overline{\bar{V}} q^{+++}\right)+\frac{(3 t+\delta(1+\gamma))^{2}}{18 t}$.

It is easy to check that $\pi_{B}^{d}=2\left(\bar{V} q^{-}+\overline{\bar{V}} q^{+}-\frac{\bar{V}}{2}\right)+\frac{(3 t+\delta \gamma)^{2}}{18 t}>\pi_{B}^{C}$, because $2\left(\bar{V} q^{-}+\overline{\bar{V}} q^{+}-\frac{\bar{V}}{2}\right)>$ $\bar{V} q^{-}+\overline{\bar{V}} q^{+}-\overline{\bar{V}} q^{+++}$when $\frac{\bar{V}}{2}<\overline{\bar{V}} \leq \frac{\bar{V}(3 t-\delta \gamma)}{3 t+\delta(1-\gamma)}$ applies (we ignore $\varepsilon$ as it is arbitrarily small). Trivially, also $\pi_{B}^{d}=2\left(\bar{V} q^{-}+\overline{\bar{V}} q^{+}-\overline{\bar{V}} q^{+++}\right)+\frac{(3 t+\delta(1+\gamma))^{2}}{18 t}>\pi_{B}^{C}$. Hence, Partial Fragmentation never occurs at equilibrium when $\frac{\bar{V}}{2}<\overline{\bar{V}} \leq \frac{\bar{V}(3 t-\delta \gamma)}{3 t+\delta(1-\gamma)}$ applies.

c. Case $\frac{\bar{V}(3 t-\delta \gamma)}{3 t+\delta(1-\gamma)}<\overline{\bar{V}}$. Because $\frac{\bar{V}(3 t-\delta \gamma)}{3 t+\delta(1-\gamma)}<\overline{\bar{V}}$ holds, we have $\bar{V} q^{-}+\overline{\bar{V}} q^{+}-\overline{\bar{V}} q^{+++}<\overline{\bar{V}} q^{-}$. Hence, the constraint on $F_{B}$ in $(8)$ is violated. Therefore, configuration $(0 A, 1 A B)$ can never occur when $\frac{\bar{V}}{2}<\overline{\bar{V}} \leq \frac{\bar{V}(3 t-\delta \gamma)}{3 t+\delta(1-\gamma)}$ applies.

Summing up. We conclude that $(0 A, 1 A B)$ can never occur at equilibrium.

II(b). Market configurations $(0 B, 1 A B),(1 B, 0 A B),(1 A, 0 A B)$

No equilibrium entailing these configurations exists. The proof is specular to that for $(0 A, 1 A B)$.

III. Market configuration $\left(0 i, 1 i^{\prime}\right) i=A, B, i^{\prime} \neq i$

The set of candidates $\left\{\mathbf{F}^{\mathbf{C}}\right\}_{T F}$ that may induce this configuration satisfies (12). We can focus only on candidate $F_{A}^{C}=F_{B}^{C}=\frac{\bar{V}}{2}$ and $\pi_{A}^{C}=\pi_{B}^{C}=\frac{\bar{V}}{2}+\frac{t}{2}$. Any other candidate in $\left\{\mathbf{F}^{\mathbf{C}}\right\}_{T F}$ is such that at least one ISP can profitably deviate by raising its fee up to $\frac{\bar{V}}{2}$, inducing the same configuration. 
a. Case $\overline{\bar{V}} \leq \frac{\bar{V}}{2}$. Consider unilateral deviations by ISP $A$. It can never profitably deviate by raising $F_{A}$ above $\frac{\bar{V}}{2}$, as both CPs would reject it. Suppose ISP $A$ deviates by decreasing $F_{A}$. Given that $F_{B}^{C}=\frac{\bar{V}}{2}$, only $(0 A, 1 A)$ can be induced. ISP $A$ can most profitably deviate setting $F_{A}^{d}=\min \left\{\overline{\bar{V}} q^{+++} ; F_{B}^{C}+\overline{\bar{V}} q^{+++}-\frac{\bar{V}}{2}-\varepsilon\right\}=\overline{\bar{V}} q^{+++}-\varepsilon$, with $\varepsilon>0$ and arbitrarily small. $A$ earns $\pi_{A}^{d}=2 \overline{\bar{V}} q^{+++}+\frac{(3 t+\delta(1+\gamma))^{2}}{18 t}$ (we ignore $\varepsilon$ as it is arbitrarily small). The deviation is unprofitable if and only if

$$
\pi_{i}^{d}=2 \overline{\bar{V}} q^{+++}+\frac{(3 t+\delta(1+\gamma))^{2}}{18 t}<\frac{\bar{V}}{2}+\frac{t}{2}=\pi_{i}^{C}
$$

Rearranging, we get that the necessary and sufficient condition for the equilibrium entailing $\left(0 i, 1 i^{\prime}\right) i=A, B, i^{\prime} \neq i$ is

$$
\overline{\bar{V}}<\left(\frac{\bar{V}}{2}-\frac{\delta(1+\gamma)(6 t+\delta(1+\gamma))}{18 t}\right) \frac{3 t}{3 t+\delta(1+\gamma)} \equiv S_{T F}
$$

b. Case $\frac{\bar{V}}{2}<\overline{\bar{V}} \leq \frac{\bar{V} 3 t}{3 t+\delta(1+\gamma)}$. Consider unilateral deviations by ISP $A$. Proceeding as in the case where $\overline{\bar{V}} \leq \frac{\bar{V}}{2}$ holds, one finds that $A$ can always deviate setting $F_{A}^{d}=$ $\min \left\{\overline{\bar{V}} q^{+++} ; F_{B}^{C}+\overline{\bar{V}} q^{+++}-\frac{\bar{V}}{2}-\varepsilon\right\}=\overline{\bar{V}} q^{+++}-\varepsilon$ and inducing $(0 A, 1 A)$. Because (19) does not hold (indeed, $2 \overline{\bar{V}} q^{+++}>\frac{\bar{V}}{2}$, when $\frac{\bar{V}}{2}<\overline{\bar{V}} \leq \frac{\bar{V} 3 t}{3 t+\delta(1+\gamma)}$ applies), no equilibrium entailing $\left(0 i, 1 i^{\prime}\right) i=A, B, i^{\prime} \neq i$ exists.

c. Case $\frac{\bar{V} 3 t}{3 t+\delta(1+\gamma)}<\overline{\bar{V}}$. Because $\frac{\bar{V} 3 t}{3 t+\delta(1+\gamma)}<\overline{\bar{V}}$, i.e. $\overline{\bar{V}} q^{+++}>\frac{\bar{V}}{2}$, holds, the necessary conditions in (12) cannot be jointly satisfied. As a result, no equilibrium entailing Total Fragmentation exists.

Summing up. We can conclude that Total Fragmentation is an equilibrium network configuration if and only if $\overline{\bar{V}}<S_{T F}$ holds. The equilibrium is such that $F_{i}^{C}=\frac{\bar{V}}{2}$ for $i=A, B$.

IV. Market configuration $\left(j \mathbf{N}, j^{\prime} A B\right)$, with $i=A, B, j, j^{\prime}=0,1$ and $j \neq j^{\prime}$

Consider $(0 N, 1 A B)$. The only equilibrium candidates respecting conditions (14) and not trivially dominated are such that $F_{A 1}^{C}=F_{B 1}^{C}=\bar{V} q^{--}$, yielding $\pi_{A}^{C}=\pi_{B}^{C}=\bar{V} q^{--}+\frac{t}{2}$. ISP $A$ can deviate setting $F_{A}^{d}=\min \left\{\frac{\bar{V}}{2}-\overline{\bar{V}} q^{+++}+F_{B}^{C} ; \frac{\bar{V}}{2}\right\}$, inducing $(0 A, 1 B)$. Because $F_{B}^{C}>\overline{\bar{V}} q^{+}$, we have $\pi_{A}^{d}=\frac{\bar{V}}{2}-\overline{\bar{V}} q^{+++}+F_{B}^{C}+\frac{t}{2}>\pi_{A}^{C}$. 
V. Market configuration $(\mathbf{0} i, \mathbf{1} i),\left(j \mathbf{N}, j^{\prime} i\right),(\mathbf{0 N}, \mathbf{1 N})$ with $i=A, B, j, j^{\prime}=0,1$ and $j \neq j^{\prime}$

None of these configurations can be an equilibrium. Indeed, by reducing its fees, the ISP carrying no CP can strictly increase profits inducing at least one CP to sign up.

\section{Proof of Proposition 3}

We follow similar steps as the Proof of Proposition 1. Using Lemma 1 (recalling that fees are discriminatory), we have profits $\pi_{j}^{*}$ of CP $j$, conditionally on connection status, described in Table 4 .

STEP 1. Using Table 4, the necessary conditions that termination fees have to satisfy for each network configuration to take place are:

1. $(0 A B, 1 A B)$

$$
F_{i j} \leq \overline{\bar{V}} q^{-}, i=A, B, j=0,1
$$

2. $(0 A, 1 A B)$

$$
\begin{array}{ll}
F_{A 0} \leq \overline{\bar{V}} q^{+}, & F_{A 1} \leq \bar{V} q^{-}+\overline{\bar{V}} q^{+}-\frac{\bar{V}}{2} \\
\overline{\bar{V}} q^{-}<F_{B 0}, & F_{B 1} \leq \bar{V} q^{-}+\overline{\bar{V}} q^{+}-\overline{\bar{V}} q^{+++}, F_{B 0} \geq F_{A 0} .
\end{array}
$$

Replace superscript 0 with superscript 1 for $(0 A B, 1 A)$.

3. $(0 B, 1 A B)$

$$
\begin{array}{ll}
F_{B 0} \leq \overline{\bar{V}} q^{+}, & F_{B 1} \leq \bar{V} q^{-}+\overline{\bar{V}} q^{+}-\frac{\bar{V}}{2} \\
\overline{\bar{V}} q^{-}<F_{A 0}, & F_{A 1} \leq \bar{V} q^{-}+\overline{\bar{V}} q^{+}-\overline{\bar{V}} q^{+++}, \quad F_{A 0}>F_{B 0} .
\end{array}
$$

Replace superscript 0 with superscript 1 for $(0 A B, 1 B)$.

4. $(0 N, 1 A B)$

$$
\overline{\bar{V}} q^{+}<F_{i 0}, \quad F_{i 1} \leq \bar{V} q^{--}, i=A, B
$$

Replace superscript 0 with superscript 1 for $(0 A B, 1 N)$. 


\begin{tabular}{|c|c|c|c|c|}
\hline $\begin{array}{l}0 \\
\tilde{0} \\
z\end{array}$ & 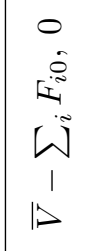 & $\begin{array}{l}F_{1}^{\vec{T}} \\
1 \\
+ \\
+ \\
\left.\right|^{+} \\
0\end{array}$ & $\begin{array}{c}\vec{D} \\
\overrightarrow{1} \\
1 \\
+ \\
+ \\
\left.\right|^{+} \\
0\end{array}$ & $\begin{array}{l}0 \\
0\end{array}$ \\
\hline$\infty$ & 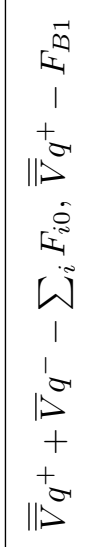 & 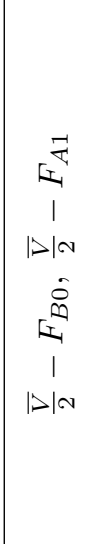 & 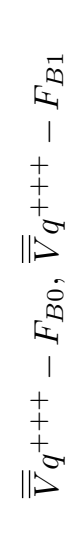 & 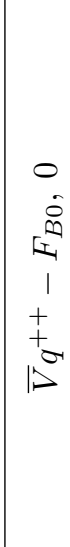 \\
\hline$\nabla$ & 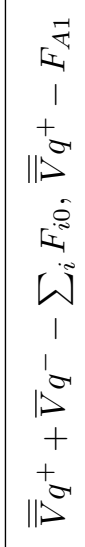 & 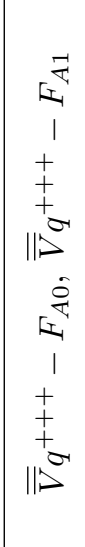 & 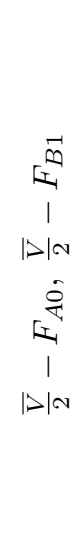 & $\mid \begin{array}{c}0 \\
0 \\
0 \\
x^{2} \\
1 \\
1 \\
+ \\
+\infty \\
10\end{array}$ \\
\hline$\frac{\infty}{3}$ & 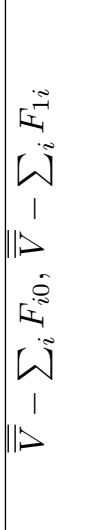 & 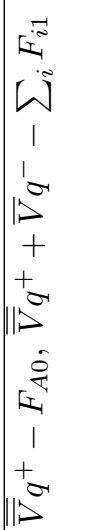 & 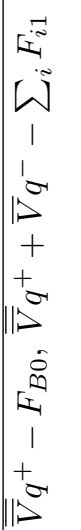 & $\begin{array}{l}\overrightarrow{\tilde{n}} \\
\tilde{\omega} \\
\dot{\omega} \\
i \\
0 \\
0\end{array}$ \\
\hline $\overrightarrow{0}$ & $\begin{array}{l}\frac{1}{2} \\
\frac{\infty}{4}\end{array}$ & $\nabla$ & $\infty$ & $\begin{array}{l}\text { Z } \\
\text { Z }\end{array}$ \\
\hline
\end{tabular}


5. $(0 A, 1 A)$

$$
\left.F_{A j} \leq \overline{\bar{V}} q^{+++}, \quad F_{B j}-F_{A j}>\frac{\bar{V}}{2}-\overline{\bar{V}} q^{+++}, \quad F_{B j}>\bar{V} q^{-}+\overline{\bar{V}} q^{+}-\overline{\bar{V}} q^{+++}, j=0,124\right)
$$

Replace subscript $A$ with subscript $B$ for configuration $(0 B, 1 B)$.

6. $(0 A, 1 B)$

$$
\begin{array}{cl}
F_{A 0} \leq \frac{\bar{V}}{2}, & \bar{V} q^{-}+\overline{\bar{V}} q^{+}-\frac{\bar{V}}{2}<F_{A 1}, \\
\bar{V} q^{-}+\overline{\bar{V}} q^{+}-\frac{\bar{V}}{2}<F_{B 0}, & F_{B 1} \leq \frac{\bar{V}}{2}, \\
F_{A 0}-F_{B 0} \leq \frac{\bar{V}}{2}-\overline{\bar{V}} q^{+++}, & -\frac{\bar{V}}{2}+\overline{\bar{V}} q^{+++} \leq F_{A 1}-F_{B 1} .
\end{array}
$$

Replace subscript $A$ with subscript $B$ for configuration $(0 B, 1 A)$.

7. $(0 N, 1 A)$

$$
\overline{\bar{V}} q^{+++}<F_{A 0}, \quad F_{A 1} \leq \bar{V} q^{++}, \quad \frac{\bar{V}}{2}<F_{B 0} \quad \bar{V} q^{--}<F_{B 1}, \quad F_{B 1}>F_{A 1} .
$$

Replace superscript 0 with superscript 1 for $(0 A, 1 N)$. Replace subscript $A$ with subscript $B$ for $(0 N, 1 B)$. Replace subscript $A$ with subscript $B$ and superscript 0 with superscript 1 for $(0 B, 1 N)$.

8. $(0 N, 1 N)$

$$
F_{i j}>\bar{V} q^{++}, \quad F_{B j}>\bar{V} q^{++}, \quad i=A, B, j=0,1 .
$$

STEP 2. This step proceeds as Step 2 of the Proof of Proposition 1.

\section{Market configuration $(0 A B, 1 A B)$}

The set of candidates $\left\{\mathbf{F}^{\mathbf{C}}\right\}_{U C}$ inducing $(0 A B, 1 A B)$ must satisfy conditions (20). An element of $\left\{\mathbf{F}^{\mathbf{C}}\right\}_{U C}$ is therefore such that $F_{i j}^{C} \leq \overline{\bar{V}} q^{-}$and $\pi_{i}^{C}=\sum F_{i j}^{C}+\frac{t}{2}$ for $i=A, B, j=0,1$.

a. Case $\overline{\bar{V}} \leq \frac{\bar{V}}{2}$. Let us focus on the candidate such that $F_{i j}^{C}=\overline{\bar{V}} q^{-}$and $\pi_{i}^{C}=2 \overline{\bar{V}} q^{-}+\frac{t}{2}$ for $i=A, B j=0,1$. As we argue below, any other candidate in $\left\{\mathbf{F}_{\mathbf{U C}}^{\mathbf{C}}\right\}$ can constitute an 
equilibrium entailing $(0 A B, 1 A B)$ only if $F_{i}^{C}=\overline{\bar{V}} q^{-}$also does. Suppose ISP $A$ deviates. Decreasing fees is never profitable. Suppose $A$ deviates by increasing $F_{A 0}$ and/or $F_{A 1}$. Because $F_{B j}^{C}=\overline{\bar{V}} q^{-}, j=0,1$, the following configurations can be induced: ${ }^{45}$

$(0 B, 1 B)$ : because $F_{B j}^{C}<\overline{\bar{V}} q^{+++}, j=0,1$, this is induced if $A$ sets $F_{A j}^{d}>$ $\max \left\{\bar{V} q^{-}+\overline{\bar{V}} q^{+}-\overline{\bar{V}} q^{+++} ; \frac{\bar{V}}{2}-\overline{\bar{V}} q^{+++}+\overline{\bar{V}} q^{-}\right\}, j=0,1$, so only conditions (24) are satisfied. ISP $A$ obtains $\pi_{A}^{d}=\frac{(3 t-\delta(1+\gamma))^{2}}{18 t}$.

$(0 A, 1 B)$ or $(0 B, 1 A)$ : ISP $A$ can induce either of these configurations in the most profitable way by setting $F_{A 0}^{d}=F_{A 1}^{d}=\frac{\bar{V}}{2}-\overline{\bar{V}} q^{+++}+\overline{\bar{V}} q^{-}$, so only conditions (25) are satisfied. ISP $A$ obtains $\pi_{A}^{d}=\overline{\bar{V}} q^{-}+\frac{\bar{V}}{2}-\overline{\bar{V}} q^{+++}+\frac{t}{2}$.

Because $\frac{(3 t-\delta(1+\gamma))^{2}}{18 t}<\pi_{A}^{C}$, the deviation inducing $(0 B, 1 B)$ is not profitable. The deviation inducing $(0 A, 1 B)$ or $(0 B, 1 A)$ is not profitable if and only if

$$
2 \overline{\bar{V}} q^{-}+\frac{t}{2} \geq \overline{\bar{V}} q^{-}+\frac{\bar{V}}{2}-\overline{\bar{V}} q^{+++}+\frac{t}{2} \Leftrightarrow \overline{\bar{V}} \geq \bar{V}\left(\frac{3 t}{6 t+\delta}\right) \equiv S_{U C}
$$

A specular reasoning applies to deviations by $B$. Hence, (28) is necessary and sufficient for candidate $F_{i j}^{C}=\overline{\bar{V}} q^{-}, i=A, B j=0,1$ to be an equilibrium entailing $(0 A B, 1 A B)$ when $\overline{\bar{V}} \leq \frac{\bar{V}}{2}$ applies.

Let us now consider the remainder of $\left\{\mathbf{F}_{\mathbf{U C}}^{\mathbf{C}}\right\}$. Following similar steps to the Proof of Proposition 1, one obtains that these candidates can constitute an equilibrium only if $F_{i j}^{C}=\overline{\bar{V}} q^{-}$does. Hence, an equilibrium entailing $(0 A B, 1 A B)$ when $\overline{\bar{V}} \leq \frac{\bar{V}}{2}$ applies exists if and only if (28) holds.

b. Case $\frac{\bar{V}}{2}<\overline{\bar{V}}$. Consider candidate $F_{i j}^{C}=\overline{\bar{V}} q^{-}, i=A, B, j=0,1$. Suppose $A$ deviates increasing $F_{A 0}^{C}$ and/or $F_{A 1}^{C}$. The following configurations can be induced:

$(0 B, 1 B)$ : this is induced when $F_{A j}^{d}>\bar{V} q^{-}+\overline{\bar{V}} q^{+}-\overline{\bar{V}} q^{+++}, j=0,1$. Only condition (24) in Step 1 are satisfied. Because $\pi_{A}^{d}=\frac{(3 t-\delta(1+\gamma))^{2}}{18 t}<\pi_{A}^{C}$, the deviation is unprofitable.

$(0 B, 1 A B)(\operatorname{resp} . \quad(0 A B, 1 B))$ : this is induced when $F_{A 1}^{d}=\bar{V} q^{-}+\overline{\bar{V}} q^{+}-\overline{\bar{V}} q^{+++}$ (resp. $\quad F_{A 0}^{d}$ ) and $F_{A 0}>\overline{\bar{V}} q^{-}$(resp. $\quad F_{A 1}>\overline{\bar{V}} q^{-}$). Only conditions (21) are satisfied. A obtains $\pi_{A}^{d}=\bar{V} q^{-}+\overline{\bar{V}} q^{+}-\overline{\bar{V}} q^{+++}+\frac{(3 t-\delta \gamma)^{2}}{18 t}$.

\footnotetext{
${ }^{45}$ Deviating by dropping a CP and inducing Partial Fragmentation is not feasible. Suppose ISP $A$ decided to deviate to $\left(j B, j^{\prime} A B\right), j=0,1, j^{\prime} \neq j$. Because, when $\overline{\bar{V}} \leq \frac{\bar{V}}{2}$ applies, $\bar{V} q^{-}+\overline{\bar{V}} q^{+}-\frac{\bar{V}}{2} \leq \overline{\bar{V}} q^{-}$ holds, the necessary condition $F_{B j} \leq \bar{V} q^{-}+\overline{\bar{V}} q^{+}-\frac{\bar{V}}{2}$ in (22) is violated. Similarly, $B$ cannot deviate to $\left(j A, j^{\prime} A B\right), j=0,1, j^{\prime} \neq j$.
} 
Using the same arguments as in the Proof of Proposition 1, it is shown that the above deviations are not profitable. Hence, there always exists an equilibrium entailing $(0 A B, 1 A B)$ when $\frac{\bar{V}}{2}<\overline{\bar{V}}$ applies.

Summing up. We can conclude that Universal Connection is an equilibrium configuration if and only if $\overline{\bar{V}} \geq S_{U C}$ holds. Any equilibrium entailing Universal Connection is such that $F_{i j}^{C} \leq \overline{\bar{V}} q^{-}$for $i=A, B$ and $j=0,1$.

\section{Market configuration $(0 A, 1 B)$}

The set of candidates $\left\{\mathbf{F}^{\mathbf{C}}\right\}_{T F}$ is such that conditions in (25) are satisfied. The proof consists in establishing that the sufficient condition for at least one of these candidates to constitute an equilibrium is weaker than (19). This is enough to prove our claim, because the latter is necessary and sufficient for Total Fragmentation to exist in the baseline model (see Proposition 1). We will focus on candidate $F_{A 0}^{C}=F_{B 1}^{C}=\frac{\bar{V}}{2}, F_{A 1}^{C}=F_{B 0}^{C}=\max \left\{\overline{\bar{V}} q^{+++} ; \bar{V} q^{-}\right\}$. Profits are such that $\pi_{A}^{C}=\pi_{B}^{C}=\frac{\bar{V}}{2}+\frac{t}{2}$.

a. Case $\overline{\bar{V}} \leq \frac{\bar{V} 3 t}{3 t+\delta(1+\gamma)}$. We will begin by describing the unilateral deviations for ISP $A$. Because $F_{B 0}^{C}=\max \left\{\overline{\bar{V}} q^{+++} ; \bar{V} q^{-}\right\}$and $F_{B 1}^{C}=\frac{\bar{V}}{2}$, the following configurations can be induced:

$(0 A B, 1 A)$ : this configuration can be induced only if $F_{B 0}^{C}=\overline{\bar{V}} q^{+++} \leq \bar{V} q^{-}+$ $\overline{\bar{V}} q^{+}-\overline{\bar{V}} q^{+++}$. A can (most profitably) deviate setting $F_{A 0}^{d}=\bar{V} q^{-}+\overline{\bar{V}} q^{+}-\frac{\bar{V}}{2}$ and $F_{A 1}^{d}=\overline{\bar{V}} q^{+}-\varepsilon$, with $\varepsilon>0$ and small. $A$ obtains $\pi_{A}^{d}=\bar{V} q^{-}+\overline{\bar{V}} q^{+}-\frac{\bar{V}}{2}+$ $\overline{\bar{V}} q^{+}+\frac{(3 t+\delta \gamma)^{2}}{18 t}$ (we ignore here $\varepsilon$ as it is arbitrarily small).

$(0 A, 1 A)$ : this configuration can be induced only if $\bar{V} q^{-}+\overline{\bar{V}} q^{+}-\overline{\bar{V}} q^{+++}<$ $\overline{\bar{V}} q^{+++}=F_{B 0}^{C}$. The most profitable deviation for $A$ inducing $(0 A, 1 A)$ is $F_{A 0}^{d}=$ $2 \overline{\bar{V}} q^{+++}-\frac{\bar{V}}{2}-\varepsilon$, with $\varepsilon>0$ and small, and $F_{A 1}^{d}=\overline{\bar{V}} q^{+++}$, yielding $\pi_{A}^{d}=$ $3 \overline{\bar{V}} q^{+++}-\frac{\bar{V}}{2}+\frac{(3 t+\delta(1+\gamma))^{2}}{18 t}$ (we ignore here $\varepsilon$ as it is arbitrarily small).

When $\overline{\bar{V}} q^{+++} \leq \bar{V} q^{-}+\overline{\bar{V}} q^{+}-\overline{\bar{V}} q^{+++}$, the relevant deviation induces $(0 A B, 1 A)$ and is not profitable if and only if

$$
\pi_{A}^{d}=\bar{V} q^{-}+\overline{\bar{V}} q^{+}-\frac{\bar{V}}{2}+\overline{\bar{V}} q^{+}+\frac{(3 t+\delta \gamma)^{2}}{18 t}<\frac{\bar{V}}{2}+\frac{t}{2}=\pi_{A}^{C} .
$$

When $\bar{V} q^{-}+\overline{\bar{V}} q^{+}-\overline{\bar{V}} q^{+++}<\overline{\bar{V}} q^{+++}$, the relevant deviation induces $(0 A, 1 A)$ and is not profitable if and only if 


$$
\pi_{A}^{d}=3 \overline{\bar{V}} q^{+++}-\frac{\bar{V}}{2}+\frac{(3 t+\delta(1+\gamma))^{2}}{18 t}<\frac{\bar{V}}{2}+\frac{t}{2}=\pi_{A}^{C} .
$$

The best deviations available to ISP $B$ are symmetric to these. Hence, the same conditions have to hold in order for them not to be profitable. Compare now the left hand side of (29) and (30) to the left hand side of (19). Noting that $\overline{\bar{V}} q^{+++}<\frac{\bar{V}}{2}$ when $\overline{\bar{V}} \leq \frac{\bar{V} 3 t}{3 t+\delta(1+\gamma)}$ applies, it is easily seen that the former two conditions are weaker than (19). This proves our claim. ${ }^{46}$

b. Case $\frac{\bar{V} 3 t}{3 t+\delta(1+\gamma)}<\overline{\bar{V}}$. As shown in the Proof of Proposition 1, no equilibrium entailing $(0 A, 1 B)$ exists in the baseline model (uniform fees) when $\frac{\bar{V} 3 t}{3 t+\delta(1+\gamma)}<\overline{\bar{V}}$. Hence, whether an equilibrium entailing $(0 A, 1 B)$ exists with discriminatory fees is of no consequence for our claim.

\section{Proof of Proposition 4}

\section{Ad platform monopoly}

We assume CPs outsource advertising to a monopolist third-party platform $K$. Hence, $K$ controls both prices $p_{0}$ and $p_{1}$ and transfers to the respective $\mathrm{CP}$ a share $\alpha \in[0,1]$ of the per impression revenue. We prove that, contrary to the baseline model, ISPs always coordinate on Universal Connection. Because, as proven in Proposition 1, this is already true in the baseline framework when $\frac{\bar{V}}{2} \leq \overline{\bar{V}}$, we will focus for brevity only on the case where $\frac{\bar{V}}{2}>\overline{\bar{V}}{ }^{47}$ The proof for the case $\frac{\bar{V}}{2} \leq \overline{\bar{V}}$ is nonetheless very similar.

The solution of stage 3 is the same as in the baseline model. As for the following stages, we proceed as follows: we first describe the outcome of Stage 2 (equilibrium profits of CPs conditional on network configuration). The output is summarized in Table 5, which is the counterpart to Table 2 for the current setup. Next, we consider Stage 1.

Stage 2. Suppose $(0 A B, 1 A B)$ is the network configuration. All advertisers multi-home if $\bar{V}+\overline{\bar{V}}-p_{0}-p_{1} \geq \bar{V}-p_{j}$, i.e. $\overline{\bar{V}} \geq p_{j}, j=0,1$. If advertisers multi-home, $K$ collects $p_{0}+p_{1} \leq 2 \overline{\bar{V}}$ total revenues. Suppose $\overline{\bar{V}}<p_{0} \leq \bar{V}$ and $/$ or $\overline{\bar{V}}<p_{1} \leq \bar{V}$. If $p_{1}>p_{0}$ (resp. $p_{0}>p_{1}$ ), all advertisers single-home on CP 0 (resp. CP 1). If advertisers single-home, $K$ collects $p_{0}$ (resp. $p_{1}$ ) in total revenues. If $\overline{\bar{V}}<p_{0}=p_{1} \leq \bar{V}$ advertisers are indifferent

\footnotetext{
${ }^{46}$ With similar steps, it can be shown that an equilibrium such that $F_{A 0}^{C}=F_{B 1}^{C}=F_{A 1}^{C}=F_{B 0}^{C}=\frac{\bar{V}}{2}$ exists if and only if (19) holds. The most profitable deviation is such that $(0 i, 1 i), i=A, B$ is induced by ISP $i$ setting $F_{i 0}^{d}=F_{i 1}^{d}=\overline{\bar{V}} q^{+++}-\varepsilon$.

${ }^{47}$ Indeed, $\max \left\{S_{U C} ; S_{T F}\right\} \leq \frac{\bar{V}}{2}$, meaning that in the baseline model, for $\frac{\bar{V}}{2} \leq \overline{\bar{V}}$ only the equilibrium entailing Universal Connection exists.
} 
between single-homing on CP 0 and single-homing on CP 1 . We assume in this case that they split equally between the two. $K$ collects then $\frac{1}{2} p_{0}+\frac{1}{2} p_{1}$. Finally, if $\bar{V}<p_{j}, \forall j, K$ collects nothing as no advertiser buys any impression. Because $\frac{\bar{V}}{2}>\overline{\bar{V}}$ by assumption, the best strategy for $K$ is to set $p_{0}^{*}=p_{1}^{*}=\bar{V}$, obtaining $\bar{V}$ total revenues (that is, $\frac{\bar{V}}{2}$ on each content). As a result, each CP obtains $\alpha \frac{\bar{V}}{2}$.

Suppose now that the network configuration is $(0 A B, 1 A)$. If an advertiser puts ads on both CPs, its surplus is $\left(\bar{V}-p_{0}\right) q^{-}+\left(\bar{V}+\overline{\bar{V}}-p_{0}-p_{1}\right) q^{+}$, given that there are $q_{B}=q^{-}$ exclusive consumers and $q_{A}=q^{+}$non-exclusive consumers. If instead the ad is put only on CP 0 , the surplus is $\bar{V}-p_{0}$, because all consumers receive a single impression. Finally, if the ad is put only on CP 1 , the advertiser's surplus is $\left(\bar{V}-p_{1}\right) q^{+}$, because only $q^{+}$consumers are impressed (once), while the remainder are not impressed at all. Because $\frac{\bar{V}}{2}>\overline{\bar{V}}$ by assumption, $K$ optimally sets $p_{0}^{*}=\bar{V}$ and $p_{1}^{*}>\overline{\bar{V}}$, inducing advertisers to single-home on CP 0, that has the largest audience. $K$ collects $\bar{V}$ total revenues as a result, CP 0 obtains $\alpha \bar{V}$ and CP 1 obtains no revenues. Similar results apply to all other network configurations involving Partial Fragmentation.

Consider now network configuration $(0 A, 1 B)$. If an advertiser puts ads on both CPs, its total surplus is $\left(\bar{V}-p_{0}\right) \frac{1}{2}+\left(\bar{V}-p_{1}\right) \frac{1}{2}$. If instead ads are put only on CP $j$, the surplus is $\left(\bar{V}-p_{j}\right) \frac{1}{2}$. Thus, with similar steps as above, platform $K$ optimally sets $p_{0}^{*}=p_{1}^{*}=\bar{V}$ and advertisers single-home. We assume advertisers split equally on the two CPs, hence revenues collected by each $\mathrm{CP}$ are equal to $\alpha \frac{\bar{V}}{2}$. The same applies to $(0 B, 1 A)$.

Consider now network configuration $(0 A, 1 A)$ or $(0 B, 1 B)$. With a reasoning similar to that of case $(0 A B, 1 A B)$, it can be proved that the equilibrium is such that $p_{0}^{*}=p_{1}^{*}=\bar{V}$, because $\frac{\bar{V}}{2}>\overline{\bar{V}}$ by assumption. Advertisers split on the two CPs and single-home. Revenues collected by each CP are $\alpha \frac{\bar{V}}{2} q^{+++}$.

Finally consider network configurations $(0 N, 1 A B)$ and $(0 N, 1 i), i=A, B$. In all such cases, $K$ optimally sets $p_{0}^{*}=p_{1}^{*}=\bar{V}$, inducing single-homing on CP 1 . Revenues collected by CP 1 are equal to $\alpha \bar{V}$ if $(0 N, 1 A B)$ and to $\alpha \bar{V} q^{++}$if $(0 N, 1 i)$ is the market configuration. Symmetrical results would be obtained with market configurations $(1 N, 0 A B)$ and $(1 N, 0 i), i=A, B$.

Table 5 summarizes the profit made by each CP, conditionally on network configuration.

Stage 1. Using Table 5 and proceeding as in the Proof of Proposition 1, it can be easily established that Universal Connection is the only equilibrium, with $F_{A}=F_{B}=\frac{\alpha \bar{V}}{4}$. 


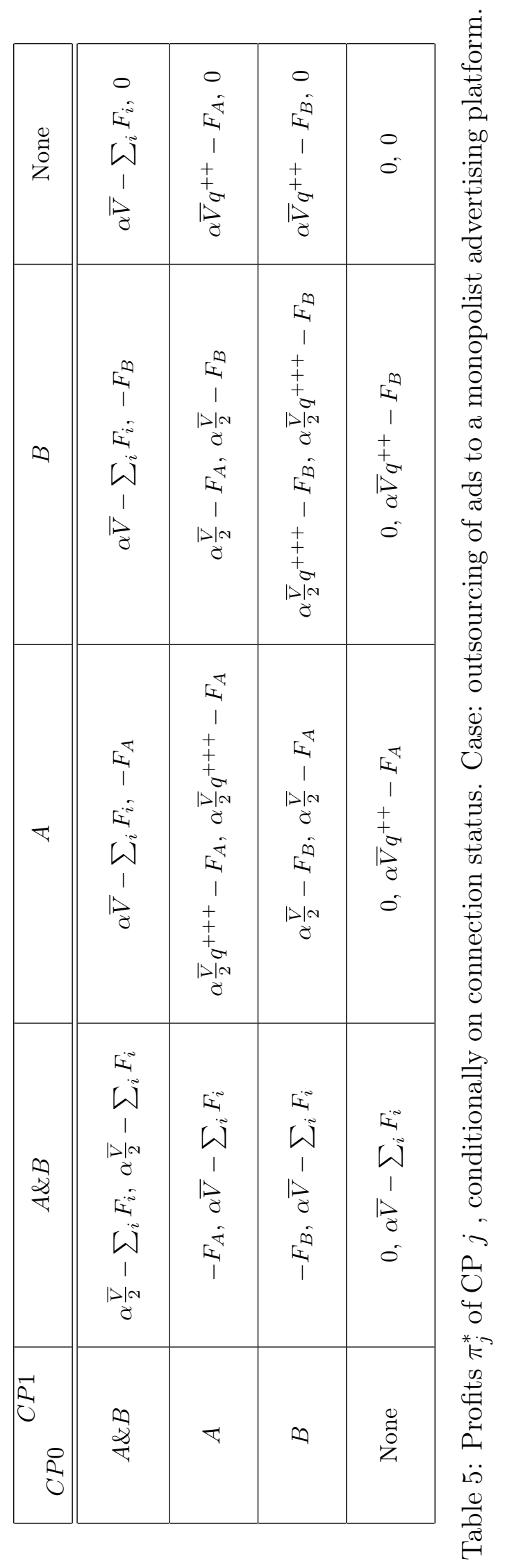




\section{Ad platform duopoly}

Consider now the case with an advertising platform duopoly. Let us denote two advertising platforms by $X$ and $Y$ and suppose CP 0 (resp. 1) outsources ad impressions to $X$ (resp. $Y$ ). Consequently, $X$ (resp. $Y$ ) controls $p_{0}$ (resp. $p_{1}$ ) and transfers to CP 0 (resp. 1) a share $\alpha \in[0,1]$ of the per impression revenue. Stage 2 of the game is identical to that of the baseline model. Thus, Lemma 1 still holds and profits of CPs (conditionally on network configuration) are as described in Table 2, with the only difference that revenues from ad impressions have to be multiplied by $\alpha .{ }^{48}$ Hence, the same equilibria as in Proposition 1 are obtained, with the only difference that values $\bar{V}$ and $\overline{\bar{V}}$ should be replaced by $\alpha \bar{V}$ and $\alpha \overline{\bar{V}}$, respectively.

\section{Proof of Proposition 5}

We assume that CPs outsource management of ad impressions to the ISPs. ISP $i=A, B$ thus posts a price $p_{i j}$ for an impression on one of its subscribers visiting $\mathrm{CP} j=0,1$. We show that no ISP has any incentive to abandon Universal Connection.

Stage 3 is as in the baseline model. Suppose the market configuration is $(0 A B, 1 A B)$ and consider stage 2 of the game. Recall that subscriber demands are $q_{i}^{*}=\frac{1}{2}, i=A, B$ with this configuration, and that consumers subscribe to only one ISP. An advertiser that wants to reach a consumer subscribing to ISP $i$ can choose to place an ad only on CP 0 (paying $p_{i 0}$ ), only on $\mathrm{CP} 1$ (paying $p_{i 1}$ ) or on both CPs (paying $p_{i 0}+p_{i 1}$ ). If an advertiser places an ad on both ISPs and both CPs, it obtains $\sum_{i=A, B}\left(\bar{V}+\overline{\bar{V}}-p_{i 0}-p_{i 1}\right) \frac{1}{2}$. If an advertiser places an ad on a single CP $j$ but on both ISPs, it obtains $\bar{V}-\left(p_{i j}+p_{i^{\prime} j}\right) \frac{1}{2}, i \neq i^{\prime}$. If an advertiser places an ad on ISP $i$ and on both CPs, it obtains $\left(\bar{V}+\overline{\bar{V}}-p_{i 0}-p_{i 1}\right) \frac{1}{2}$. Finally, if an advertiser places an ad only on ISP $i$ and only on CP $j$, it obtains $\left(\bar{V}-p_{i j}\right) \frac{1}{2}$. Assume that $\frac{\bar{V}}{2} \leq \overline{\bar{V}}$. ISP $i$ sets $p_{i 0}^{*}=p_{i 1}^{*}=\frac{\overline{\bar{V}}}{2}$, so advertisers multi-home on both CPs. Assume now that $\frac{\bar{V}}{2}>\overline{\bar{V}}$. ISP $i$ can set $p_{i j}^{*}=\bar{V}$ and $p_{i j^{\prime}}^{*}>\bar{V}$, thereby inducing all advertisers that want to reach its subscribers to place ads only on CP $j$. ISP $i$ gains $\frac{\bar{V}}{2}$ on the advertising market: this is the same as the termination fee it would get with Total Fragmentation (see the Proof of Proposition 1). It follows that no ISP can increase profits by deviating from $(0 A B, 1 A B) .{ }^{49}$ With similar reasoning, it can be shown that no other equilibrium exists.

\footnotetext{
${ }^{48}$ For example, profits of CPs in case $(0 A B, 1 A B)$ are $\pi_{j}^{*}=\alpha \overline{\bar{V}}-\sum_{i=A, B} F_{i}$, profits in the case $(0 A, 1 B)$ are $\pi_{0}^{*}=\alpha \frac{\bar{V}}{2}-F_{A}$ and $\pi_{1}^{*}=\alpha \frac{\bar{V}}{2}-F_{B}$, and so on.

${ }^{49}$ The same outcome would result with a different but equally plausible assumption on how the ISP sells impressions. Suppose that ISP $i=A, B$ makes a take-it-or-leave-it offer to an advertiser consisting in two ad impressions per consumer (i.e. one on each $\mathrm{CP}$ the consumer visits) at a per impression price $p_{i}$. If an
} 


\section{References}

[1] Ambrus, A., Calvano, E. and Reisinger, M., 2013. Either or Both Competition: a "TwoSided" Theory of Advertising With Overlapping Viewerships. Working Paper available at https://www.econstor.eu/dspace/bitstream/10419/79912/1/VfS_2013_pid_326.pdf.

[2] Ambrus, A. and Reisinger, M., 2006. Exclusive vs Overlapping Viewers in Media Markets. SFB/TR 15 Discussion Paper No. 161.

[3] Anderson, S.P., Foros, Ø. and Kind, H. J., 2014. Competition for Advertisers and for Viewers in Media Markets. Mimeo.

[4] Armstrong, M., 2006. Competition in Two-Sided Markets . RAND Journal of Economics, 37, 668-691.

[5] Athey, S., Calvano, E. and Gans, J.S., 2013. The Impact of the Internet on Advertising Markets for News Media. Rotman School of Management Working Paper No. 2180851.

[6] Bourreau, M., Kourandi, F. and Valletti, T.M., 2015. Net Neutrality with Competing Internet Platforms. The Journal of Industrial Economics, 63, 30-73.

[7] Caillaud, B. and Jullien, B., 2003. Chicken \& Egg: Competition among Intermediation Service Providers. RAND Journal of Economics, 34, 309-328.

[8] Calvano, E. and Jullien, B., 2012. Issues in online advertising and competition policy: a two-sided market perspective, Recent Advances in the Analysis of Competition Policy and Regulation. Edited by J.E. Harrington and Y. Katsoulacos, Edward Elgar Publishing.

[9] Choi, J.P., 2010. Tying in Two-Sided Markets with Multi-Homing. Journal of Industrial Economics, 58, 607-626.

[10] Choi, J.P., Jeon, D.S. and Kim, B.C., forthcoming. Net Neutrality, Business Models, and Internet Interconnection. American Economic Journal: Microeconomics.

advertiser puts ads only on ISP $i=A, B$, it obtains $\left(\bar{V}+\overline{\bar{V}}-2 p_{i}\right) \frac{1}{2}$. If the advertiser places ads on both platforms, it obtains $\sum_{i=A, B}\left(\bar{V}+\overline{\bar{V}}-2 p_{i}\right) \frac{1}{2}$. Advertisers thus buy ads on ISP $i$ if and only if $p_{i} \leq \frac{\bar{V}+\overline{\bar{V}}}{2}$, $i=A, B$. As a result, the equilibrium price is $p_{i}^{*}=\frac{\bar{V}+\overline{\bar{V}}}{2}, i=A, B$, inducing all advertisers to multi-home. This means that even with Universal Connection, ISPs are able to extract the entire advertiser surplus and have no reason to deviate. 
[11] Choi, J.P. and Kim, B.C., 2010. Net neutrality and investment incentives. RAND Journal of Economics, 41, 446-471.

[12] de Cornière, A. and Taylor, G., 2014 Integration and search engine bias. RAND Journal of Economics, 45(3), 576-597, 09

[13] Economides, N. and Hermalin, B., 2012. The Economics of Net Neutrality. RAND Journal of Economics, 43, 602-629.

[14] Economides, N. and Tag J., 2012. Net Neutrality on the Internet: A two-sided market analysis. Information Economics and Policy, 24, 91-104.

[15] European Commission, 2009. Telecoms Commission launches case against UK over privacy and personal data protection. Available at http://europa.eu/rapid/pressrelease_IP-09-570_en.htm?locale=en.

[16] FCC, 2015. In the Matter of Protecting and Promoting the Open Internet. Available at http://transition.fcc.gov/Daily_Releases/Daily_Business/2015/db0312/FCC15-24A1.pdf.

[17] Gal-Or, E. and Dukes, A., 2003. Minimum Differentiation in Commercial Media Markets. Journal of Economics \& Management Strategy, 12, 291-325.

[18] Hermalin, B.E. and Katz, M.L., 2007. The economics of product-line restrictions with an application to the Net Neutrality debate. Information Economics and Policy, 19, $215-248$.

[19] Kourandi, F., Krämer, J. and Valletti, T.M., 2015. Net Neutrality, Exclusivity Contracts and Internet Fragmentation. Information System Research, 26, 320-338.

[20] Krämer J., Wiewiorra, L. and Weinhardt, C., 2013. Net Neutrality: a Progress Report. Telecommunications Policy, 37, 794-813.

[21] Lee, R.S. and Wu, T., 2009. Subsidizing creativity through network design: Zero-pricing and Net Neutrality. Journal of Economic Perspectives, 23, 61-76.

[22] Musacchio, J., Schwartz,G., and Walrand, J., 2009. A two-sided market analysis of provider investment incentives with an application to the net-neutrality issue. Review of Network Economics, 8, 22-39. 
[23] Njoroge, P., Ozdaglar, A., Stier-Moses, N.E., and Weintraub, G.Y., 2013. Investment in Two Sided Markets and the Net Neutrality Debate. Review of Network Economics, 12, 355-402.

[24] Rey, P. and Tirole, J., 2007. A Primer on Foreclosure. Handbook of Industrial Organization, Vol. 3, ed. Mark Armstrong and Robert H. Porter, 2145-2220. Amsterdam: North-Holland.

[25] Rey, P. and Vergé, T., 2008. Economics of Vertical Restraints. Handbook of Antitrust Economics. Paolo Buccirossi (ed.), The MIT Press, 9, 353-390.

[26] Rochet, J.C. and Tirole, J., 2006. Two-sided markets: a progress report. RAND Journal of Economics, 37, 645-667.

[27] Schuett, F., 2010. Net Neutrality: A Survey of the Economic Literature. Review of Network Economics, 9, Article 1.

[28] Waterman, D. and Choi, S., 2011. Non-discrimination rules for ISPs and vertical integration: Lessons from cable television. Telecommunications Policy, 35, 970-983. 